OPEN ACCESS

Edited by:

María Serrano,

Miguel Hernández University of Elche,

Spain

Reviewed by:

Dawei Zhang,

Sichuan University, China

Eri Ogiso-Tanaka,

National Museum of Nature and Science, Japan

Shucai Wang,

Linyi University, China

*Correspondence:

Qing-Yao Shu

qyshu@zju.edu.cn

Specialty section:

This article was submitted to

Crop and Product Physiology,

a section of the journal

Frontiers in Plant Science

Received: 18 March 2021

Accepted: 10 May 2021

Published: 11 June 2021

Citation:

Li R-Q, Jiang M, Huang J-Z, Møller IM and Shu Q-Y (2021)

Mutations of the Genomes

Uncoupled 4 Gene Cause ROS

Accumulation and Repress

Expression of Peroxidase Genes

in Rice. Front. Plant Sci. 12:682453.

doi: $10.3389 / f p / s .2021 .682453$

\section{Mutations of the Genomes Uncoupled 4 Gene Cause ROS Accumulation and Repress Expression of Peroxidase Genes in Rice}

\author{
Rui-Qing Li1,2, Meng Jiang', Jian-Zhong Huang ${ }^{1,3}$, lan Max Møller ${ }^{4}$ and Qing-Yao Shu ${ }^{1,3 *}$ \\ ${ }^{1}$ Hainan Institute of Zheijang University, Yazhou Bay Science and Technology City, Sanya, China, ${ }^{2}$ College of Agronomy, \\ Anhui Agricultural University, Hefei, China, ${ }^{3}$ National Key Laboratory of Rice Biology, Institute of Crop Sciences, Zhejiang \\ University, Hangzhou, China, ${ }^{4}$ Department of Molecular Biology and Genetics, Aarhus University, Slagelse, Denmark
}

The Genomes Uncoupled 4 (GUN4) is one of the retrograde signaling genes in Arabidopsis and its orthologs have been identified in oxygenic phototrophic organisms from cyanobacterium to higher plants. GUN4 is involved in tetrapyrrole biosynthesis and its mutation often causes chlorophyll-deficient phenotypes with increased levels of reactive oxygen species (ROS), hence it has been speculated that GUN4 may also play a role in photoprotection. However, the biological mechanism leading to the increased ROS accumulation in gun4 mutants remains largely unknown. In our previous studies, we generated an epi-mutant allele of OsGUN4 (gun4 ${ }^{e p i}$ ), which downregulated its expression to $\sim 0.5 \%$ that of its wild-type (WT), and a complete knockout allele gun41 due to abolishment of its translation start site. In the present study, three types of $\mathrm{F}_{2}$ plant derived from a gun4-1/gun4epi cross, i.e., gun4-1/gun4-1, gun4-1/gun4epi and gun4 $4^{e p i}$ gun $4^{e p i}$ were developed and used for further investigation by growing them under photoperiodic condition ( $16 \mathrm{~h} / 8 \mathrm{~h}$ light/dark) with low light (LL, $100 \mu \mathrm{mol}$ photons $\left.\mathrm{m}^{-2} \mathrm{~s}^{-1}\right)$ or high light $\left(\mathrm{HL}, 1000 \mu \mathrm{mol}\right.$ photons $\left.\mathrm{m}^{-2} \mathrm{~s}^{-1}\right)$. The expression of OsGUN4 was light responsive and had two peaks in the daytime. gun4-1/gun4$1-\mathrm{F}_{2}$ seeds showed defective germination and died within 7 days. Significantly higher levels of ROS accumulated in all types of OsGUN4 mutants than in WT plants under both the $L L$ and $H L$ conditions. A comparative RNA-seq analysis of WT variety LTB and its gun $4^{e p i}$ mutant HYB led to the identification of eight peroxidase (PRX)-encoding genes that were significantly downregulated in HYB. The transcription of these eight $P R X$ genes was restored in transgenic HYB protoplasts overexpressing OsGUN4, while their expression was repressed in LTB protoplasts transformed with an OsGUN4 silencing vector. We conclude that OsGUN4 is indispensable for rice, its expression is light- and oxidative-stress responsive, and it plays a role in ROS accumulation via its involvement in the transcriptional regulation of $P R X$ genes.

Keywords: GUN4, reactive oxygen species, anti-oxidative response, peroxidase, rice 


\section{INTRODUCTION}

The Genomes Uncoupled 4 (GUN4) gene is one of six GUN genes that are involved in retrograde signaling pathways (Larkin et al., 2003). The gun mutants were originally identified in Arabidopsis thaliana as signal transduction mutants that uncouple their nuclear gene expression from chloroplast development (Susek et al., 1993). GUN4 is highly conserved in oxygenic photosynthetic plant species (Strand et al., 2003; Formighieri et al., 2012; Brzezowski et al., 2014; Li et al., 2017). GUN4 binds the product and substrate of Mg-chelatase, an enzyme that produces $\mathrm{Mg}$-Proto, and activates $\mathrm{Mg}$-chelatase, hence plays a regulatory role in chlorophyll biosynthesis in Arabidopsis thaliana (Larkin et al., 2003; Peter and Grimm, 2009; Adhikari et al., 2011; Richter et al., 2016), Chlamydomonas reinhardtii (Brzezowski et al., 2014), Synechocystis sp. (Sobotka et al., 2008; Chen et al., 2015) and rice (Zhou et al., 2012; Li et al., 2014a; Jiang et al., 2019).

Genomes Uncoupled 4 is not an essential Mg-chelatase subunit in vitro or in vivo and hence is not essential for chlorophyll biosynthesis (Larkin et al., 2003; Davison et al., 2005; Verdecia et al., 2005; Peter and Grimm, 2009), but all gun4 mutants have reduced chlorophyll contents throughout their life cycle. Indeed, mutations of GUN4 and its orthologs not only resulted in reduced chlorophyll biosynthesis, but also greatly altered the content of tetrapyrrole intermediates. In the rice mutant $\mathrm{HYB}$ with OsGUN4 being significantly downregulated, the content of heme and protoporphyrin IX (PPIX) was significantly increased while that of other tetrapyrrole metabolites such as Mg-protoporphyrin IX (Mg-PPIX) was significantly decreased (Li et al., 2017). Similar increases in PPIX content were reported for gun4 mutants of Synechocystis sp. (Sobotka et al., 2008) and C. reinhardtii (Formighieri et al., 2012), while little or no increase was reported for Arabidopsis (Mochizuki et al., 2008).

An additional phenotype of gun4 mutants is their increased photosensitivity, which has since led to the suggestion that GUN4 plays a role in photoprotection (Larkin et al., 2003; Adhikari et al., 2011; Formighieri et al., 2012). Larkin et al. (2003) proposed that GUN4 - the $\mathrm{H}$ subunit of Mg-Chelatase $(\mathrm{ChlH})$ complexes could envelop and thereby shield PPIX and Mg-PPIX from collisions with $\mathrm{O}_{2}$ that might yield reactive oxygen species (ROS), and hence contribute to photoprotection. In contrast, Adhikari et al. (2011) developed gun4 mutants with altered binding ability to porphyrin and investigated their ROS content, and demonstrated that the porphyrin-binding activity of GUN4 did not help attenuate ROS production. In C. reinhardtii, Brzezowski et al. (2014) proposed that GUN4 functions in photoprotection by preventing PPIX and Mg-PPIX from interacting with oxygen and thereby decreasing ROS production based on their study on the gun 4 and chlD-1 mutants. However, Tabrizi et al. (2016) demonstrated that purified GUN4 together with oxidatively damaged $\mathrm{ChlH}$ increased the rate of PPIX-generated singlet oxygen $\left({ }^{1} \mathrm{O}_{2}\right)$ production in the light by a factor of 5 and 10 and therefore concluded that protoporphyrin bound to GUN4 was the actual ${ }^{1} \mathrm{O}_{2}$ singlet oxygen generator. Also, in C. reinhardtii gun4 mutants, Formighieri et al. (2012) observed perturbations in electron transport with a strongly decreased PSI-to-PSII ratio, which was accompanied by an enhanced activity of the plastid terminal oxidase (PTOX). They therefore proposed that GUN4 plays a physiological role in decreasing photosystem II excitation pressure. Thus, that the functioning mechanism of GUN4 in photoprotection and ROS homeostasis is still uncertain.

In plant chloroplasts, PSI and PSII reactive centers are the major sources of ROS (Asada, 1999; Møller et al., 2007), which in turn can damage the photosystems and disrupt electron transfer chain (ETC) (Laloi et al., 2004). One of the efficient anti-oxidant systems in the chloroplast involves enzymatic systems to remove ROS once formed (Dietz, 2016). ROS and redox regulation play important roles in the regulation of photosynthesis (Dietz and Hell, 2015). Two types of peroxidases (PRXs), the thiolbased and ascorbate-dependent peroxidases, are employed to link detoxification of peroxides and serve roles in redox regulation and retrograde signaling within and from the chloroplast (Dietz, 2016).

Obviously, the increased ROS accumulation observed in gun4 mutants could also result from either increased production or decreased removal, or both. However, most studies on gun 4 mutants have focused on the binding ability of GUN4 to porphyrins and its role in ROS production. The possibility that GUN4 also plays a role in the regulation of ROS response genes such as PPX genes has never been addressed.

We identified an antioxidant response element (ARE) in the promoter of OsGUN4 and demonstrated that its methylation dramatically downregulated its expression in the xantha mutant HYB, which produced a chlorophyll-deficient phenotype (Li et al., 2014a). We further demonstrated that the ${ }^{1} \mathrm{O}_{2}$ level was significantly reduced in HYB compared with that of its wild-type (WT) parent Longtepu B (LTB) (Li et al., 2017). These observations led us to propose that GUN4 plays a role in the regulation of ROS response genes, which may explain the increased ROS accumulation in gun4 mutants. In the present study, we first examined the response of OsGUN4 expression to light intensity and oxidative stress and assessed ROS accumulation in osgun4 mutants; we then performed RNA-seq and identified differentially expressed PRX genes and finally; we tested the effect of OsGUN4 on the expression of PRX genes in protoplasts by silencing and complementing OsGUN4. We show that OsGUN4 expression is light- and oxidative-stress responsive, and OsGUN4 plays a role in ROS accumulation via its involvement in the transcriptional regulation of $P R X$ genes in rice.

\section{MATERIALS AND METHODS}

\section{Plant Materials}

From wild-type indica variety Longtepu B (LTB), we previously developed a xantha mutant line, Huangyu B (HYB) via gamma ray mutagenesis (Zhou et al., 2006). HYB carries an epi-mutant allele of OsGUN4 (hereafter gun4 ${ }^{e p i}$ ), which downregulates the expression of OsGUN4 to 0.5\% that of LTB (Li et al., 2014a). Further, through gamma ray irradiation, our previous study also succeeded in generating and identifying a few OsGUN4 mutant alleles in the background of a japonica line GS113 by 
crossing HYB to GS113 $\mathrm{M}_{2}$ plants (Li et al., 2014a). Among them, the gun4- 1 allele is a complete knockout mutation because its translation initiation codon (ATG) is completely deleted (and thus no OsGUN4 protein is expected to be translated) (Li et al., 2014a). In the present study, the $\mathrm{F}_{2}$ seeds of the $\mathrm{F}_{1}$ hybrid plant of GS113 (gun4-1)/HYB (gun $4^{e p i}$ ) were used for various investigations, together with LTB and HYB (Supplementary Figure 1).

For easy management, germinated seeds were first grown in plastic trays with soils taken from paddy fields (surface, not sterilized) of Zhejiang University Experimental Farm at $30^{\circ} \mathrm{C}$ for 7 days in growth rooms with LED (light emitting diode) under $16 \mathrm{~h} / 8 \mathrm{~h}$ light/dark, at low light (LL, $100 \mu \mathrm{mol}$ photons $\mathrm{m}^{-2} \mathrm{~s}^{-1}$ ) or high light (HL, $1000 \mu \mathrm{mol}$ photons $\mathrm{m}^{-2} \mathrm{~s}^{-1}$ ) conditions. For assessing the effect of light intensity on seedling growth and gene expression, seven-day old seedlings grown on soil were transferred to $1 \times$ MS liquid medium (Murashige and Skoog, 1962) and grown under either LL or HL conditions for another 7-28 days. For assessment of $\mathrm{H}_{2} \mathrm{O}_{2}$ effect, seedlings that had been grown on soil (7 days) and $1 \times$ MS medium (21 days) under LL were transferred to $1 \times$ MS liquid medium with or without $1 \mathrm{mM}$ $\mathrm{H}_{2} \mathrm{O}_{2}$ and grown for another 7 days under LL. Liquid medium was replaced every 3 days.

For genotyping of the $\mathrm{F}_{2}$ seedlings, genomic DNA was extracted from leaf segments and subject to high resolution melting (HRM) curve analysis with primers of $5^{\prime}$-TTACCGG CAGGCCGACGAGA-3' and 5'-TGCCCAGGAGCTGTGTCC CT-3' according to Tan et al. (2016).

For investigation of diurnal expression of OsGUN4, seedlings of LTB and Nipponbare (japonica) were grown in soil under HL conditions ( $16 \mathrm{~h} / 8 \mathrm{~h}$ light/dark) for 35 days after germination (DAG), and their leaves were sampled every $2 \mathrm{~h}$ for RNA extraction within a 24 -h period.

\section{Quantification of Chlorophylls and Tetrapyrrole Metabolites}

The concentrations of chlorophylls were determined using $0.3 \mathrm{~g}$ fresh leaf tissues following a published method (Peter and Grimm, 2009). For measurement of PPIX, Mg-PPIX and protochlorophyllide (pchlide), $0.3 \mathrm{~g}$ fine powder of liquid nitrogen-ground leaves were immediately mixed with precold alkaline acetone containing $0.1 \mathrm{~N} \mathrm{NH} 4 \mathrm{OH}$ (9:1; v/v), and centrifugated at $16,000 \mathrm{~g}$ for $5 \mathrm{~min}$. Subsequently, the supernatants were collected for extraction of PPIX (Papenbrock and Grimm, 2001), Mg-PPIX (Papenbrock and Grimm, 2001), and pchlide (Koski and Smith, 1948). The contents of PPIX, MgPPIX and pchlide were determined with commercial enzymelinked immunosorbent assay (ELISA; Engvall and Perlmann, 1971) kits (Jiangsu Jingmei Biotechnology Co., Ltd., Yancheng, China) following the manufacturer's instructions.

\section{Measurement of Singlet Oxygen, Hydrogen Peroxide and Total ROS}

The concentration of singlet oxygen $\left({ }^{1} \mathrm{O}_{2}\right)$ was determined using the SOSG (singlet oxygen sensor green) method (Hideg et al., 2002). Briefly, the liquid nitrogen homogenized power of $0.3 \mathrm{~g}$ leaf samples were suspended with pre-cold lysis buffer (GENMED Scientifics Inc., Shanghai, China), and then immediately centrifuged at $4^{\circ} \mathrm{C}$ for $10 \mathrm{~min}(5000 \mathrm{~g})$. Subsequently, SOSG (GENMED Scientifics Inc.) was added to the supernatant to a final concentration of $10 \mu \mathrm{M}$. The fluorescence spectra were detected at excitation of $485 \mathrm{~nm}$ and emission of $520 \mathrm{~nm}$ using a fluorescence spectrophotometer (359S, Lengguang Tech., Shanghai, China).

The hydrogen peroxide $\left(\mathrm{H}_{2} \mathrm{O}_{2}\right)$ content was measured according to the method of Rokebul Anower et al. (2017) with minor modifications. $0.3 \mathrm{~g}$ leaf tissues were ground in liquid nitrogen and then immediately mixed with pre-chilled KrebsRinger phosphate $(145 \mathrm{mM} \mathrm{NaCl}, 5.7 \mathrm{mM}$ sodium phosphate, $4.86 \mathrm{mM} \mathrm{KCl}, 0.54 \mathrm{mM} \mathrm{CaCl}_{2}, 1.22 \mathrm{mM} \mathrm{MgSO}_{4}, 5.5 \mathrm{mM}$ glucose, $\mathrm{pH}$ 7.35). Subsequently, the homogenate was centrifuged at $12,000 \mathrm{~g}$ for $20 \mathrm{~min}$ at $4^{\circ} \mathrm{C}$, and then the supernatants were incubated with $50 \mu \mathrm{M}$ Amplex ${ }^{\circledR}$ Red reagent (10-acetyl-3,7dihydrophenoxazine, Thermo Fisher Scientific, Shanghai, China) and $0.1 \mathrm{U} / \mathrm{mL}$ horseradish peroxidase (HRP, Thermo Fisher Scientific, Shanghai China) at $37^{\circ} \mathrm{C}$ for $10 \mathrm{~min}$ away from light. The fluorescence was measured at $530 \mathrm{~nm}$ under irradiation of $590 \mathrm{~nm}$ light for excitation by using a VersaFluor Fluorometer (Bio-Rad, Hercules, CA, United States) and calculated based on a standard curve constructed using known concentrations of $\mathrm{H}_{2} \mathrm{O}_{2}$.

The total ROS concentration was determined according to the method of Joo et al. (2005) with modifications. In brief, for assessment of ROS in seedlings, the above-ground part of seedlings was weighed, quickly frozen and ground into powder in liquid nitrogen, and immediately suspended in $10 \mathrm{mM}$ Tris$\mathrm{HCl}$ buffer ( $\mathrm{pH}$ 7.3) for extraction. The mixture was centrifuged at $16,000 \mathrm{~g}$ for $5 \mathrm{~min}$, and after collecting the supernatant, the pellet was suspended with buffer and centrifuged for another $5 \mathrm{~min}$. For assessment of ROS in protoplasts, the transfected protoplasts containing $4 \times 10^{4}$ protoplasts/ $\mu \mathrm{L}$ were added with $10 \mathrm{mM} \mathrm{H} \mathrm{H}_{2}$ DCFDA (Thermo Fisher Scientific, Waltham, MA, United States) at $4^{\circ} \mathrm{C}$ in DMSO.

The supernatant was used for ROS measurement by adding $10 \mathrm{mM} \mathrm{H} \mathrm{H}_{2}$ DCFDA (Thermo Fisher Scientific, Waltham, MA, United States) at $4^{\circ} \mathrm{C}$ in DMSO. The fluorescence was measured at $530 \mathrm{~nm}$ under irradiation of $488 \mathrm{~nm}$ light for excitation by using a VersaFluor fluorometer (Bio-Rad, Hercules, CA, United States) with six biological replicates. The ROS content, expressed as relative fluorescence units, was obtained from six successive measurements and is given as units per milligram of fresh weight.

\section{Quantitative Real-Time PCR Analysis}

Total RNA was extracted from fresh leaves or protoplasts using the Qiagen Spin Plant RNA Mini Kit (Qiagen, Hilden, Germany). cDNA was reverse transcribed from $1 \mu \mathrm{g}$ total RNA with oligo- $\mathrm{dT}_{18}$ Primer using GoScript ${ }^{\mathrm{TM}}$ Reverse Transcription System Kit (Promega, WI, United States) according to the manufacturer's instructions. Quantitative real-time PCR (qRTPCR) was performed using a SYBR Green GoTaq ${ }^{\circledR}$ qPCR Master Mix containing ROX as internal control (Promega, WI, United States). All qRT-PCRs used the following cycling 
conditions: $10 \mathrm{~min}$ at $95^{\circ} \mathrm{C}, 40 \mathrm{cycles}$ of $30 \mathrm{~s}$ at $94^{\circ} \mathrm{C}, 30 \mathrm{~s}$ at $55^{\circ} \mathrm{C}$ and $60 \mathrm{~s}$ at $72^{\circ} \mathrm{C}$. Relative mRNA abundance was analyzed with the rice Ubiquitin gene as internal reference using the $2^{-\Delta \Delta \mathrm{Ct}}$ method (Livak and Schmittgen, 2001). Primers used for qRT-PCR are listed in Supplementary Table 1.

\section{RNA Sequencing}

Total RNA of LTB and HYB was extracted from fresh leaf tissues of 35 DAG seedlings using the Qiagen Spin Plant RNA Mini Kit (Qiagen, Hilden, Germany). cDNA libraries were constructed with oligo- $\mathrm{dT}_{18}$ Primer using GoScript ${ }^{\mathrm{TM}}$ Reverse Transcription System Kit (Promega, WI, United States) according to the manufacturer's instructions, and were subsequently sequenced on an Illumina Hiseq 2000 platform (Beijing Novogene Bioinformatics Technology Co., Ltd., Beijing, China). For mapping, the raw reads were cleaned by removing adapter sequences using the Cutadapt (v1.11) software and then aligned to the reference genome sequences (IRGSP-1.0 $0^{1}, 09 / 17 / 2016$ ) by using the Tophat v2.0.9 program with $E$-value $<10^{-5}$ as cutoff point (Trapnell et al., 2012). For detection of differentially expressed genes (DEGs), the DESeq package (ver 2.1.0) was used with a false discovery rate (FDR) $\leq 0.05$ and the absolute value of the $\log _{2}$ (fold change $\geq 2$ ) with $R P K M \geq 1$ as the threshold to determine significant differences of gene expression. Gene ontology (GO) enrichment was conducted according to PANTHER Overrepresentation Test (released 20210224) by using GO Ontology database (released 02/01/2021) using Oryza sativa database as a reference list, and was visualized with ggplot2 $\mathrm{R}$ package. KEGG pathways were analyzed with a FDR $\leq 0.05$ as significant levels of differential expression. DEGs potentially related to ROS homeostasis were identified and subjected to RT-q PCR analysis using gene-specific primers (Supplementary Table 1).

\section{Functional \\ Complementation/Overexpression and Silencing of Genes in a Protoplast System}

For construction of OsGUN4 complementary expression vector, a DNA fragment encompassing the full open reading frame of OsGUN4 and its upstream (2447 bp) and downstream (518 bp) sequences were amplified from genomic DNA of Nipponbare using primers OGUN4F and OGUN4R (Supplementary Table 1), which was cloned into a pUCm-T vector (Sangon Biotech, Shanghai, China). The recombinant pUCm-T vector was then digested with NcoI/HindIII and the target fragment was cloned into binary vector pCAMBIA 1301 using the same restriction enzymes. The ultimate vector is named pOsGUN4-C.

For construction of OsPRX39 overexpression vector, the full open reading frame of OsPRX39 (999 bp) sequences were amplified from genomic DNA of Nipponbare with the primers OPRX39F/OPRX39R (Supplementary Table 1), which was cloned into a pUCm-T vector (Sangon Biotech, Shanghai, China),

\footnotetext{
${ }^{1} \mathrm{ftp}$ //ftp.ensemblgenomes.org/pub/plants/release-32/fasta/oryza_sativa/dna/ Oryza_sativa.IRGSP-1.0.dna.toplevel.fa.gz
}

and the recombinant vector was digested with $N c o I / E c o R I$. Subsequently, the target fragment was simultaneously cloned into the digested binary vector of pCAMBIA 1301 (by using NcoI/EcoRI), the OsPRX39 overexpression vector was thus constructed and named p1301-Ubi-PRX39.

For silencing of OsGUN4, the hairpin RNA (hpRNA) downregulating system was used and a silencing vector was constructed according to $\mathrm{Li}$ et al. (2014b). An OsGUN4 fragment (+562 to +761 bp downstream of the $\left.{ }^{+1} \mathrm{ATG}\right)$, containing part of the coding sequence and a partial $3^{\prime}$ UTR was amplified using primers RGUN4F and RGUN4R (Supplementary Table 1) and cloned into a pUCm-T vector. Two fragments containing the OsGUN4 fragment were cloned from the recombinant pUCm-T vector by double restriction with NotI/BamHI and XhoI/KpnI, respectively. They were then sequentially ligated into pBSSK-IN in the sense and antisense orientations. The OsGUN4 hairpin structure in the recombinant pBSSK-IN was introduced into pCAMBIA1301-35S using restriction enzymes KpnI and SacI. The recombinant pCAMBIA1301-35S vector is named pOsGUN4-I.

Using the slightly modified enzymolysis method (Zhang et al., 2011), protoplasts of LTB and HYB were extracted from 35 DAG seedlings grown under LL conditions and used for transient expression analysis. They were transformed with the OsGUN4 complementation/overexpression or silencing vectors with PEGmediated transformation according to Bart et al. (2006). Briefly, $100 \mu \mathrm{L}$ solution containing $4 \times 10^{4}$ protoplasts $/ \mu \mathrm{L}$ was transformed with $10 \mu \mathrm{g}$ plasmids and incubated in W5 solution $\left(2.4 \times 10^{4}\right.$ protoplasts/ $\left.\mu \mathrm{L}\right)$ in darkness for about $1 \mathrm{~h}$. The transformed protoplasts were then cultured at low light or high light for another $6 \mathrm{~h}$ before RNA extraction.

\section{Statistical Analysis}

All experiments were performed with six biological replicates. Values are expressed as means \pm standard deviations. Comparisons of data from different groups were analyzed using ANOVA test followed by the Tukey's Multiple Comparison Test with $P<0.05$.

\section{RESULTS}

\section{OsGUN4 Expression Is Responsive to Light Intensity and Oxidative Stress}

To detect the daily variation of OsGUN4 expression, we first we investigated its relative expression in seedlings of indica variety LTB and japonica variety Nipponbare at different times during a 24-h period (Figure 1A). We observed that the expression of OsGUN4 was lower at night and quickly increased after day-break at $0600 \mathrm{~h}$. Two peaks of expression were observed at 1000 and $1400 \mathrm{~h}$ for both varieties. The expression gradually decreased in the afternoon and remained at a low level at night. These results suggest that the expression of OsGUN4 had the nature of diurnal oscillation, and the relatively reduced expression at the mid-day suggests that its expression is also subject to internal physiological changes. 


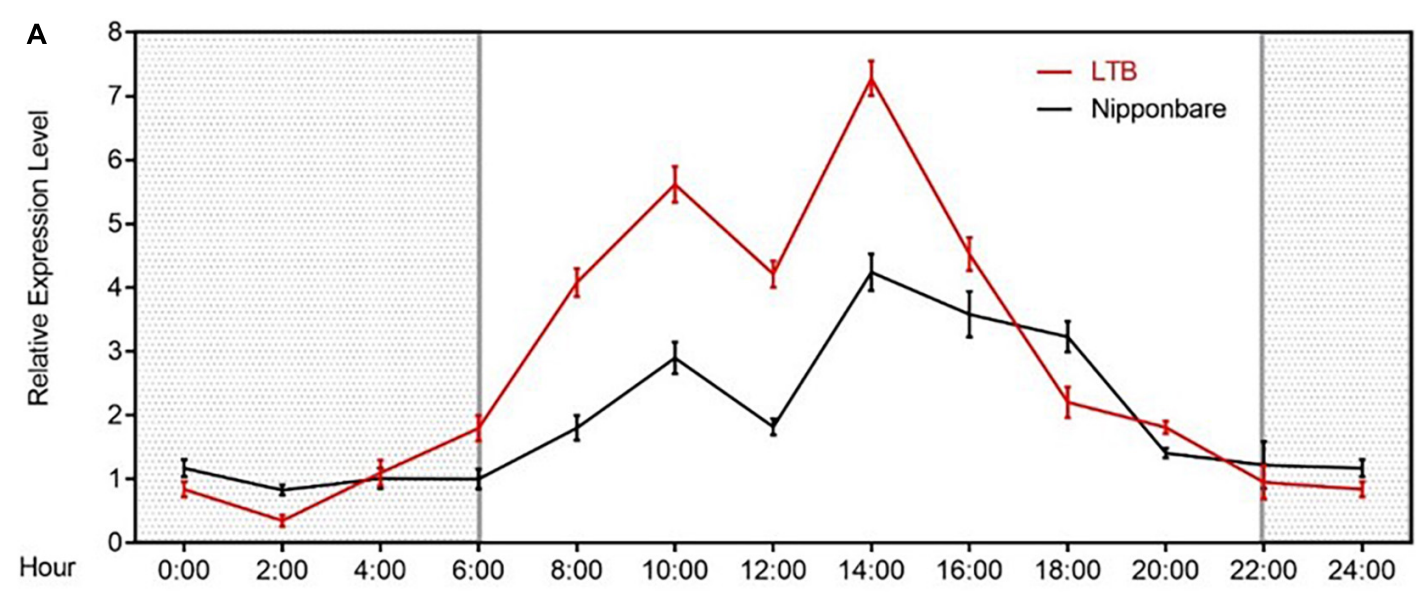

B

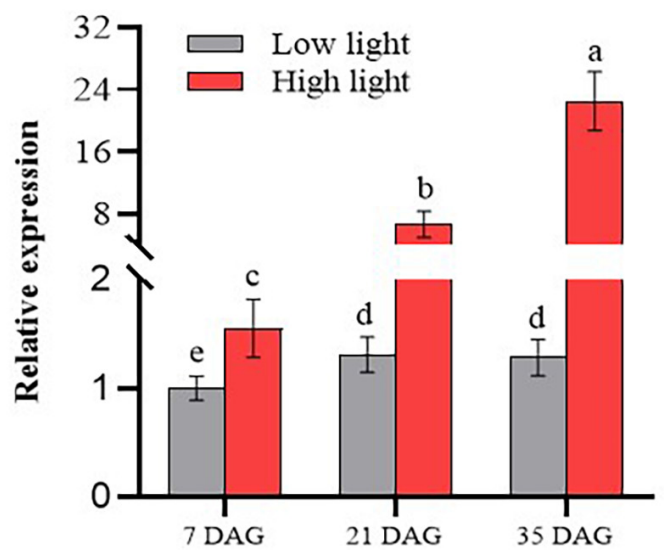

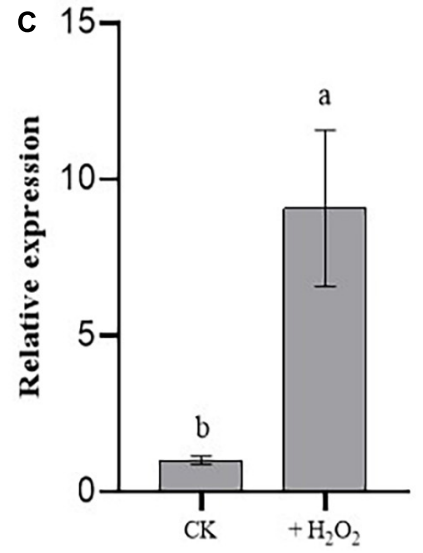

FIGURE 1 | Relative expression of OsGUN4 in seedlings 35 days after germination. (A) Variation of OsGUN4 expression in wild-type indica rice Longtepu B (LTB) and japonica rice Nipponbare across a 24-h period grown in growth room in photoperiodic conditions (16 h light/8 h dark). The light intensity was $1000 \mu \mathrm{mol} \mathrm{m} \mathrm{m}^{-2} \mathrm{~s}^{-1}$ and the temperature was set at a constant $30^{\circ} \mathrm{C}$. Samples were taken every $2 \mathrm{~h}$ from 6:00 am when light was turned on. Relative expression is reported relative to that of Nipponbare at 6:00 am, which was assigned a value of 1. (B) Relative expression of OsGUN4 in LTB seedlings at 35 days after germination (DAG) grown under either high light (1000 $\left.\mu \mathrm{mol} \mathrm{m} \mathrm{m}^{-2} \mathrm{~s}^{-1}\right)$ or low light $\left(100 \mu \mathrm{mol} \mathrm{m}^{-2} \mathrm{~s}^{-1}\right)$ with temperature set at a constant $30^{\circ} \mathrm{C}$. Relative expression is reported in relative to that of LTB seedlings at 7 DAG, which was assigned a value of 1. (C) Relative expression of OsGUN4 in LTB seedlings subjected to oxidative stress. Seedlings of 7 DAG were first grown in $1 \times$ MS liquid medium for 21 days and then further grown with or without 1 mM $\mathrm{H}_{2} \mathrm{O}_{2}$ for another 7 days. All seedlings were under low light conditions. Relative expression is reported in relative to that of that control, which was assigned a value of 1.

To examine the responsiveness of OsGUN4 to light intensity, the abundance of OsGUN4 mRNA transcripts was examined for seedlings grown under LL and HL conditions at 7, 21, and 35 days after germination (DAG) (Figure 1B). When grown under LL conditions, the expression of OsGUN4 was low and hardly increased with plant age (Figure 1B and Supplementary Table 2). Under HL conditions, the OsGUN4 expression level significantly increased with seedling age, and was much higher than that of seedlings grown under LL conditions at all ages (Figure 1B). For example, at $7 \mathrm{DAG}$, the OsGUN4 expression level in seedlings under HL conditions was 1.53 that of seedlings under LL, while at $35 \mathrm{DAG}$, the relative value increased to 17.49. Thus, OsGUN4 expression was responsive to light intensity and increased with seedling age.

To examine its responsiveness to oxidative stress, the expression of OsGUN4 was examined in seedlings grown for
7 days in medium with $1 \mathrm{mM} \mathrm{H}_{2} \mathrm{O}_{2}$. The OsGUN4 expression level was significantly higher (8.8-fold) in seedlings treated with $\mathrm{H}_{2} \mathrm{O}_{2}$ than in the control plants (Figure 1C and Supplementary Table 2), indicating a response to oxidative stress.

\section{Impaired Growth of the OsGUN4 Mutant Seedlings}

Down-regulation of OsGUN4 is known to result in chlorophyll deficiency and reduced plant growth, but it was not known whether rice could survive a complete OsGUN4 knockout under different light conditions. During the germination stage, we observed that a high percentage of $F_{2}$ seeds derived from the gun4-1/gun4 $4^{e p i} \mathrm{~F}_{1}$ plants could not develop into complete seedlings and died within 7 DAG (Figure 2 and Supplementary Figure 2A). 


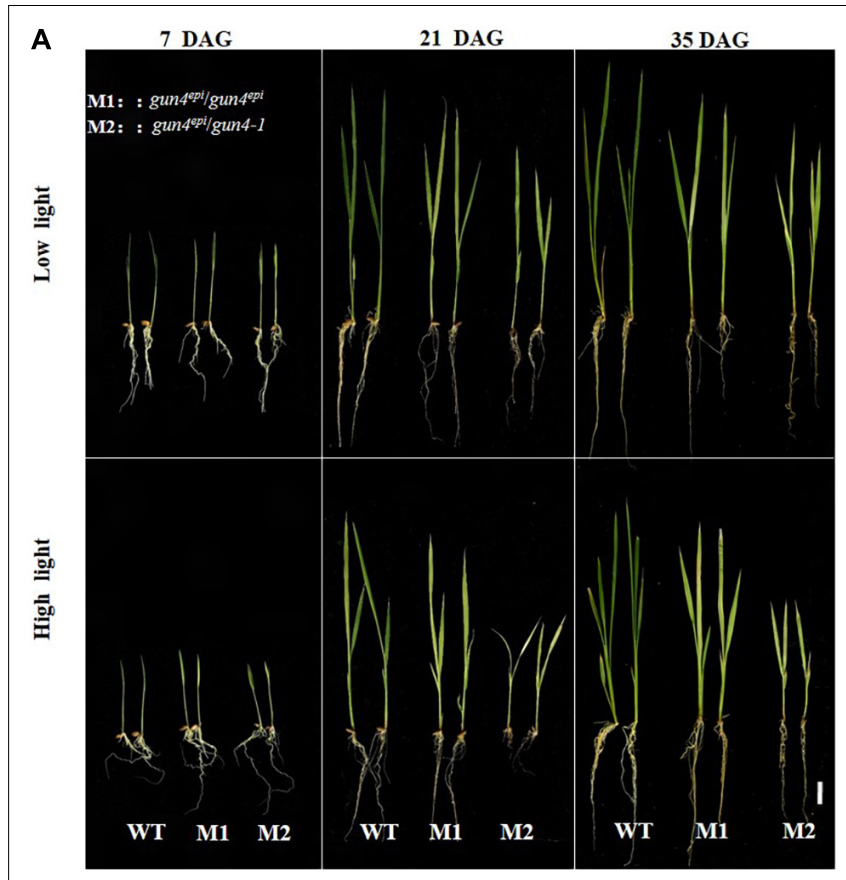

B

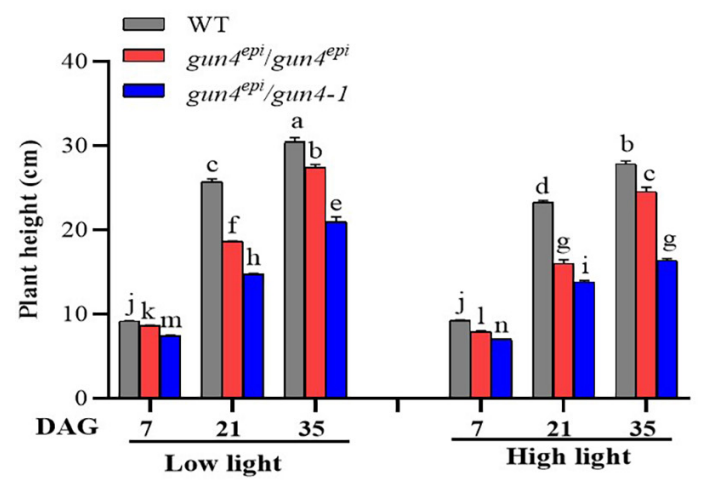

FIGURE 2 | Effects of high light on growth of the OsGUN4 mutants. (A) The phenotype and (B) plant height of wild-type (WT; c.v. LTB) and $F_{2}$ seedlings derived from gun $4^{e p i}$ /gun $4-1 \mathrm{~F}_{1}$ plants at 7,21 , and 35 days after germination (DAG). Values are means $\pm \mathrm{SD}(n=6)$ and analyzed for significant differences by two-way ANOVA followed by the Tukey's multiple comparison test,

$P<0.05$. Scale bar $=3 \mathrm{~cm}$. For the background information of the $F_{1}$ plants, see Supplementary Figure 1.

To distinguish the effect of the gun $4^{e p i}$ and gun4-1 mutations on seed germination and seedling growth, we performed an HRM-based genotyping (Supplementary Figure 1B). It was revealed that all the early dying germinated seeds belonged to the gun4-1/gun4-1 genotype, suggesting OsGUN4 is indispensable for rice seedling growth. The gun $4^{e p i} / g u n 4^{e p i}$ and $g u n 4^{e p i} / g u n 4-$ $1 \mathrm{~F}_{2}$ seedlings were further grown under different conditions and their growth and ROS content were measured.

Most $\mathrm{F}_{2}$ seedlings with the gun $4^{e p i} /$ gun $4-1$ genotype survived, but their growth was significantly slower and weaker than the WT and $g u n 4^{e p i} / g u n 4^{e p i}$ ones, particularly under the HL conditions (Figure 2A; Supplementary Figure 2B; and Supplementary Table 3). Under HL conditions, the gun $4^{e p i} / g u n 4-1 \mathrm{~F}_{2}$ seedlings were significantly shorter than the gun $4^{e p i} / g u n 4^{e p i}$ ones (Figure 2B), suggesting that there is a dosage effect of the $g u n 4^{e p i}$ allele on seedling growth.

\section{Changes in Tetrapyrrole Metabolite Levels}

To assess the effect of OsGUN4 mutations on tetrapyrrole metabolism, the concentrations of chlorophyll (Chl) and three key tetrapyrrole metabolites was measured in 35 DAG seedlings grown under LL and HL conditions (Figure 3 and Supplementary Table 4). Under LL conditions, in consistent with phenotypical changes, although the concentration of Chl and tetrapyrrole metabolites was significant lower in the OsGUN4 mutants than the WT, the differences were very limited between mutants and the WT, with the maximum difference being observed for Chl ( $<30 \%$, Figure 3D); No significantly differences were detected for any of the metabolites between the $\mathrm{F}_{2}$ seedlings of $g u n 4^{e p i} / g u n 4^{e p i}$ and $g u n 4^{e p i} / g u n 4-1$ (Figure 3).

Under HL condition, the metabolite concentration differences became much more pronounced between the two mutants and the WT (Figure 3). PPIX is the tetrapyrrole metabolite that can be diverted into both heme (through Fe-branch) and Chl (through Mg-branch), its concentration was significantly higher $(+60 \%)$ in the two mutants than in the WT (Figure 3A). However, the metabolites in the $\mathrm{Mg}$-branch were significantly reduced in both mutants (Figures 3B-D). The two mutants had $89 \%$ of the MgPPIX concentration in the WT (Figure 3B). The reduction of pchlide and $\mathrm{Chl}$ contents was more dramatic; the $\mathrm{F}_{2}$ seedlings of gun $4^{e p i} / g u n 4^{e p i}$ and gun $4^{e p i} / g u n 4-1$ had pchlide content only 63 and $56 \%$ that of the WT, and Chl content only 65 and $41 \%$ that of the WT, respectively (Figures 3C,D).

When WT seedlings were moved from LL to HL conditions, the concentration of PPIX, Mg-PPIX and Chl was significantly reduced, while that of pchlide significantly increased. In the two OsGUN4 mutants, the concentrations of all metabolites except PPIX significantly decreased (Figure 3). Thus, OsGUN4 mutations also changed the responsiveness of tetrapyrrole metabolism to light intensity.

\section{Elevated Accumulation of ROS in OsGUN4 Mutant Seedlings}

To further examine whether the repressive effect of OsGUN4 mutations on seedling growth and tetrapyrrole metabolism was ROS-dependent, we measured the concentrations of total ROS, $\mathrm{H}_{2} \mathrm{O}_{2}$ and ${ }^{1} \mathrm{O}_{2}$ in the $\mathrm{F}_{2}$ and WT seedlings at different growth stages under LL and HL conditions. Significantly greater total ROS and $\mathrm{H}_{2} \mathrm{O}_{2}$ contents, but lower ${ }^{1} \mathrm{O}_{2}$ content, was observed in the mutant $\mathrm{F}_{2}$ seedlings than the WT line, irrespective of the growth conditions and mutant genotype (Figure 4 and Supplementary Table 5).

For 7 DAG seedlings, we were able to measure the total ROS level in all three types of mutant seedlings (Figure 4A) and observed that the total ROS level in the gun4-1/gun4-1 seedlings, irrespective of growth condition, was much higher than those in the WT and other two mutants. For example, under HL conditions, gun4-1/gun4-1 $\mathrm{F}_{2}$ seedlings accumulated 

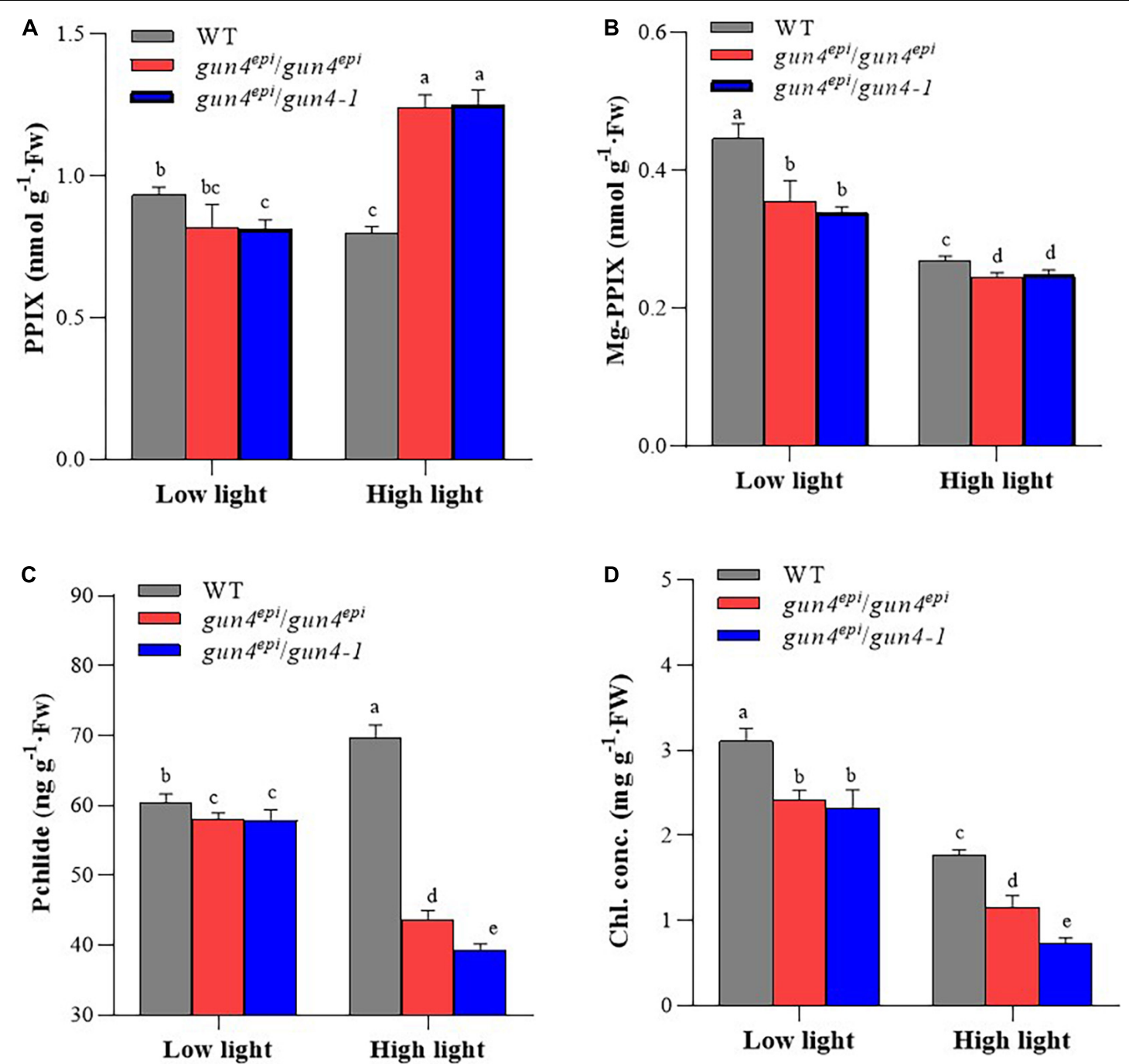

FIGURE 3 | The concentration of tetrapyrrole metabolites in wild-type (WT) and OsGUN4 mutant rice seedlings. Seedlings were at 35 days after germination (DAG) grown under low (100 $\mu \mathrm{mol} \mathrm{m} \mathrm{m}^{-2} \mathrm{~s}^{-1}$ photons) or high (1000 $\mu \mathrm{mol} \mathrm{m} \mathrm{m}^{-2} \mathrm{~s}^{-1}$ photons) light conditions. WT, wild-type variety Longtepu B, gun4epi/gun4 $4^{e p i}$ and gun4 $4^{e i}$ /gun4-1 are $\mathrm{F}_{2}$ seedlings derived from the gun4epi/gun4-1 $\mathrm{F}_{1}$ plants. PPIX, protoporphyrin IX; Mg-PPIX, Mg-protoporphyrin IX; Pchlide, protochlorophyllide; $\mathrm{Chl}$, chlorophyll. Values are means $\pm \mathrm{SD}(n=6)$ and analyzed for significant differences by two-way ANOVA followed by the Tukey's multiple comparison test, $P<0.05$. For the background information of the $F_{1}$ plants, see Supplementary Figure 1.

9.18, 3.54, and 2.97 times as much total ROS than WT, $g u n 4^{e p i} / g u n 4^{e p i}$ and gun $4^{e p i} / g u n 4-1$, respectively (Figure 4A). Because gun4-1/gun4-1 $\mathrm{F}_{2}$ seedlings did not survive beyond 7 DAG, ROS content could only be measured on seedlings of the other two mutants at 21 and 35 DAG. The gun $4^{\text {epi }} / g u n 4$ $1 \mathrm{~F}_{2}$ seedlings accumulated significantly higher levels of total ROS than the gun $4^{e p i} / g u n 4^{e p i}$ ones. Comparatively, both types of mutant seedlings contained far more ROS than the WT at all time point, i.e., the $g u n 4^{e p i} / g u n 4^{e p i} \mathrm{~F}_{2}$ seedlings accumulated 2.7-3.3 times, under the LL conditions, and 2.6-3.5 times under the HL conditions (Figure 4A) more ROS than the WT (Figure 4A), respectively.

The total ROS level in the gun $4^{e p i} / g u n 4-1 \quad \mathrm{~F}_{2}$ seedlings increased with seedling age under both the LL and HL conditions. A similar trend was observed in $g u n 4^{e p i} / g u n 4^{e p i} \mathrm{~F}_{2}$ seedlings grown under the HL conditions (Figure 4A). At a given growth stage, significantly more ROS accumulated in seedlings grown under HL conditions than under LL conditions irrespective of genotype (Figure 4A).

To differentiate different types of ROS, the seedlings at 35 DAG were further assessed for the level of $\mathrm{H}_{2} \mathrm{O}_{2}$ and ${ }^{1} \mathrm{O}_{2}$. Significantly higher levels of $\mathrm{H}_{2} \mathrm{O}_{2}$ were observed in mutant seedlings than that in the WT; the differences became more significant under $\mathrm{HL}$ conditions. The levels of $\mathrm{H}_{2} \mathrm{O}_{2}$ in $g u n 4^{e p i} / g u n 4^{e p i}$ and gun $4^{e p i} / g u n 4-1 \quad \mathrm{~F}_{2}$ seedlings were 2.5and 3.2-fold those of the WT, respectively (Figure $4 \mathbf{B}$ and Supplementary Table 6). Both $g u n 4^{e p i} / g u n 4^{e p i}$ and $g u n 4^{e p i} / g u n 4$ $1 \mathrm{~F}_{2}$ seedlings accumulated significantly lower levels of ${ }^{1} \mathrm{O}_{2}$ (66-87\%) compared to the WT, irrespective of growth conditions (Figure 4C and Supplementary Table 6). Furthermore, HL 

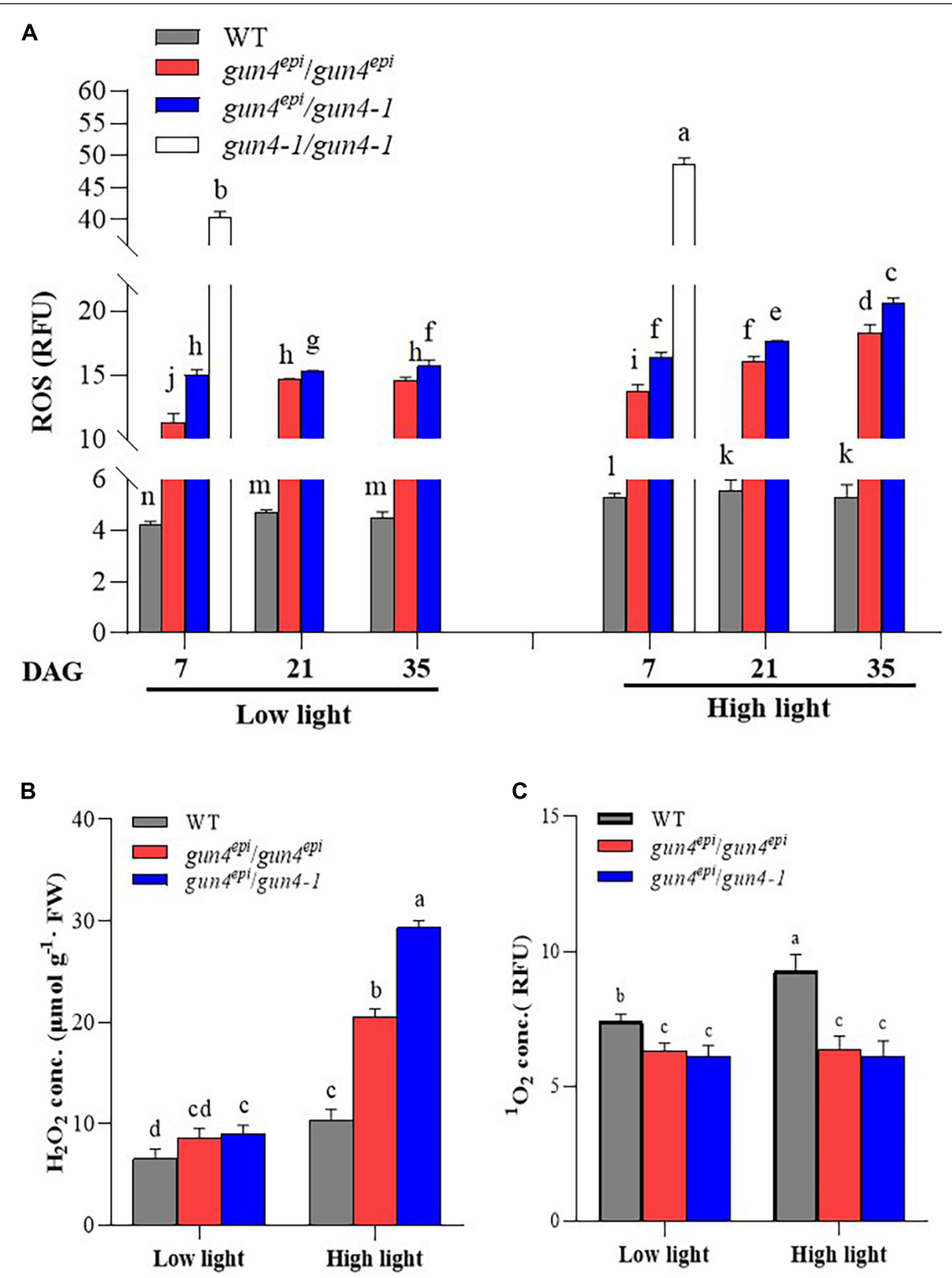

FIGURE 4 | Effects of high light on the accumulation of reactive oxygen species (ROS) in the OsGUN4 mutants. (A) The total reactive oxygen species (ROS) at 7, 21, and 35 days after germination (DAG), as well as concentrations of $\mathbf{( B )}$ hydrogen peroxide $\left(\mathrm{H}_{2} \mathrm{O}_{2}\right)$ and $\mathbf{( C )}$ singlet oxygen $\left({ }^{1} \mathrm{O}_{2}\right)$ in rice seedlings at 35 DAG. WT,

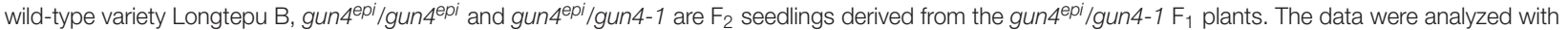
two-way ANOVA followed by the Tukey's multiple comparison test, $P<0.05$. For the background information of the $F_{1}$ plants, see Supplementary Figure 1.

treatment induced an increase in ${ }^{1} \mathrm{O}_{2}$ content by $25 \%$ in WT, while no significant change was detected in the mutants under any condition (Figure 4C).

\section{Identification of Differentially Expressed Genes Encoding ROS-Degrading Enzymes by Transcriptome Sequencing}

To examine the effect of the gun $4^{e p i}$ mutation on overall gene expression, the transcriptomes of LTB and its epigenetic mutant $\mathrm{HYB}$ at 35 DAG were sequenced (Supplementary Tables 7,8 ). Besides, a total of 468 differentially expressed genes (DEGs) were identified between HYB and LTB, with 203 genes being up-regulated and 265 down-regulated in HYB. GO analysis showed genes involved in peroxidase activity, response to oxidative stress were down-regulated (Figure 5). Further examination revealed that eight peroxidase (PRX)-encoding genes were among the down-regulated genes.

\section{Transcription of $8 P R X$ Genes Is Responsive to Oxidative Stress in WT but Significantly Repressed in OsGUN4 Mutants}

We already demonstrated that transcription of OsGUN4 is responsive to high $\mathrm{HL}$ conditions and $\mathrm{H}_{2} \mathrm{O}_{2}$ supplement 


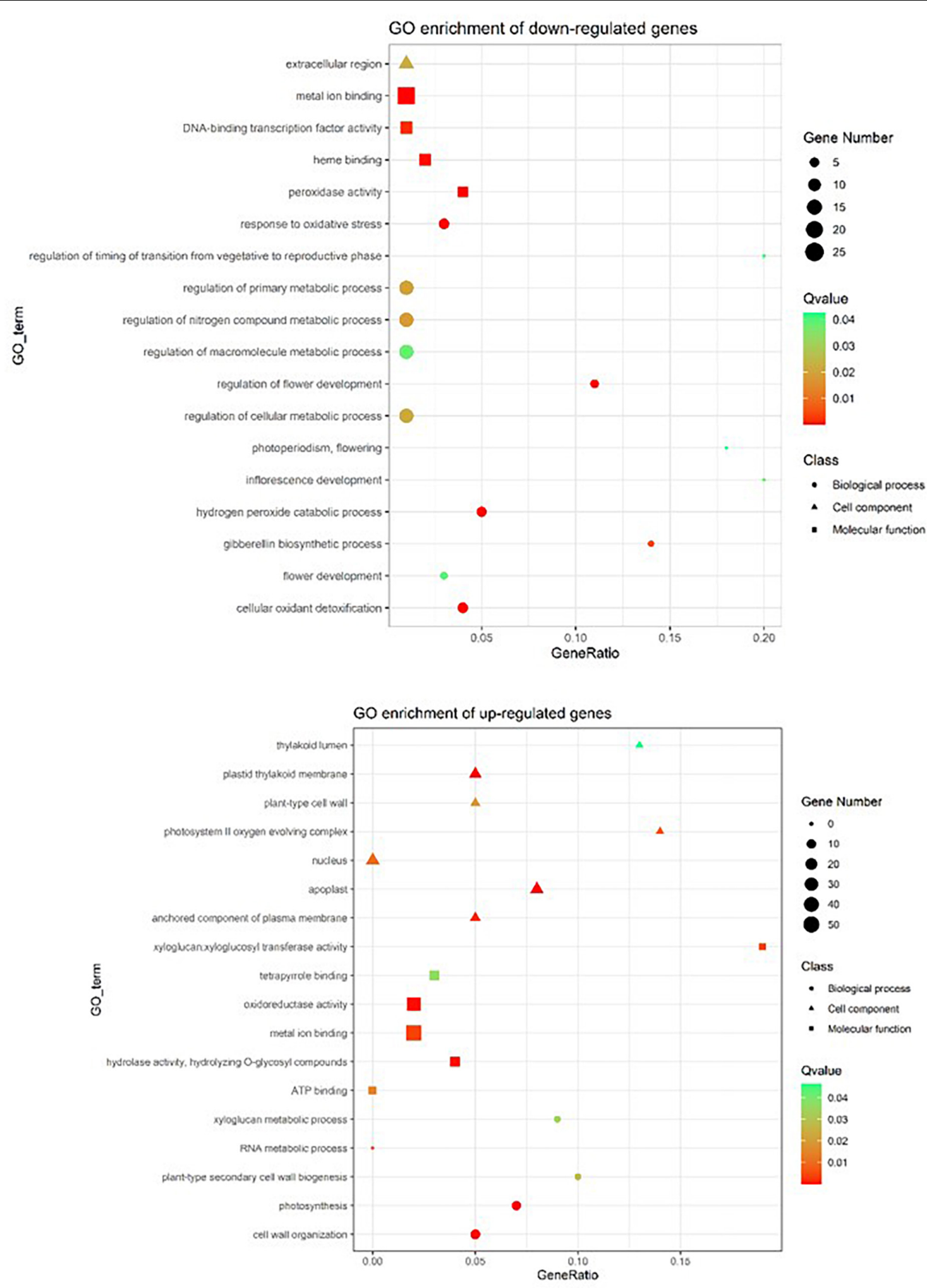

FIGURE 5 | GO enrichment of differentially expressed genes in the epigenetic gun4 mutant HYB as compared with its wild-type parent LTB, revealed by RNA-seq in 35 DAG leaves. Gene ontology (GO) enrichment was conducted according to PANTHER Overrepresentation Test (released 20210224) by using GO Ontology database (released 02/01/2021) using Oryza sativa database as a reference list. False Discovery Rate was applied for statistics. The ggplot2 was used to provide visualizations for enrichment results. 
(Figures 1B,C), this type of response of OsGUN4 expression is no longer present in HYB seedlings: OsGUN4 expression is extremely low irrespective of treatment, and no significant differences were observed for OsGUN4 transcript abundance in $\mathrm{HYB}$ and the two $\mathrm{F}_{2}$ mutant seedlings (Figure 6A). These results indicated that the OsGUN4 mutations not only dramatically reduced its transcription but also abolished its response to oxidative stresses caused by $\mathrm{HL}$ and $\mathrm{H}_{2} \mathrm{O}_{2}$. Similar performance of the mutant $\mathrm{F}_{2}$ seedlings to $\mathrm{HYB}$ indicated that the mutation effects were independent of genetic background.

qRT-PCR analyses demonstrated that the 8 PRX genes downregulated in HYB were all responsive to $\mathrm{HL}$ and exogenous $\mathrm{H}_{2} \mathrm{O}_{2}$ treatment in the WT (Figure 6 and Supplementary Table 9). The shift from LL to $\mathrm{HL}$ resulted in significant increases of transcripts abundance, i.e., from 2.96-times (OsPRX89, Figure 6H) to 8.54-times (OsPRX86, Figure 6G); Exogenous $\mathrm{H}_{2} \mathrm{O}_{2}$ treatment also generated similar increases (but slightly less than HL shift).

The transcriptional response of the 8 PRX genes to $\mathrm{HL}$ and $\mathrm{H}_{2} \mathrm{O}_{2}$ treatment seemed to be either significantly reduced or abolished in HYB and the mutant $\mathrm{F}_{2}$ seedlings (Figures 6B-I). Three genes (OsPRX-L, OsPRX 11 and OsPRX39) were no longer responsive to $\mathrm{HL}$ and $\mathrm{H}_{2} \mathrm{O}_{2}$ treatment (Figures 6B-D),
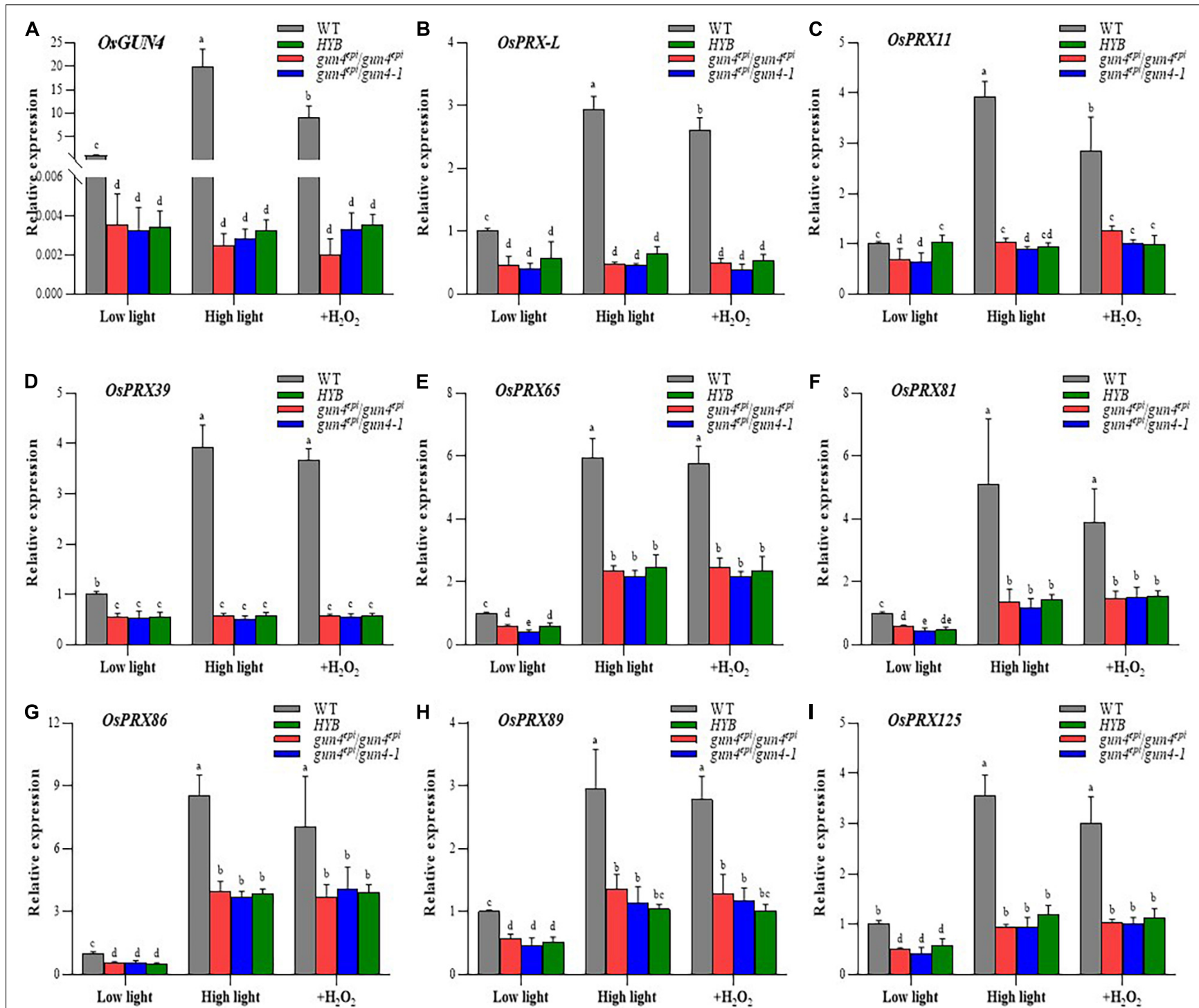

FIGURE 6 | Relative expression levels of OsGUN4 and 8 peroxidase-encoding genes. Relative expression levels of OsGUN4 (A) and 8 peroxidase (PRX)-encoding genes (B-I) in 35-day-old seedlings. WT, wild-type variety LTB; HYB, the epigenetic OsGUN4 mutant LTB (gun4 ${ }^{\text {epi }} /$ gun4 ${ }^{\text {epi }}$ ) of LTB; gun4 ${ }^{\text {epi }} /$ gun4 ${ }^{\text {epi }}$ and gun4epi/gun4-1 are $\mathrm{F}_{2}$ seedlings derived from the gun4epi/gun4-1 $\mathrm{F}_{1}$ plants. Seedlings were grown under low (100 $\mu \mathrm{mol} \mathrm{m}^{-2} \mathrm{~s}^{-1} \mathrm{photons}$ or high (1000 $\mu \mathrm{mol} \mathrm{m} \mathrm{m}^{-2} \mathrm{~s}^{-1}$ photons) light conditions, or in medium with $1 \mathrm{mM}$ exogenous $\mathrm{H}_{2} \mathrm{O}_{2}\left(+\mathrm{H}_{2} \mathrm{O}_{2}\right)$ under low light condition. Expression levels are reported relative to that of the WT grown under low light, which was assigned a value of 1 . Values are means \pm SD $(n=6)$ and analyzed for significant differences by two-way ANOVA followed by the Tukey's multiple comparison test, $P<0.05$. For more detail of seedling growth, see M\&M. 
and the remaining genes became significantly less responsive (Figures 6E-I).

\section{Silencing and Complementation of OsGUN4 Expression and Their Effect on Transcription of 8 PRX Genes in a Protoplast System}

To test whether the expression differences of the 8 PRX genes in WT and mutant seedlings are truly due to OsGUN4 mutation, LTB protoplasts were transformed with the OsGUN4 interference vector pOsGUN4-I and HYB protoplasts were transformed with the OsGUN4 complementary vector pOsGUN4-C. The transformed protoplasts were subjected to LL and HL treatment and transcription of OsGUN4 and the 8 PRX genes were then analyzed by q RT-PCR.

The expression pattern of OsGUN4 in protoplasts seemed similar to that in seedlings, i.e., its expression was extremely low in HYB and did not increase after HL treatment, in sharp contrast to what was observed in WT (Figure 7A). Importantly, we succeeded in repressing OsGUN4 expression by transforming LTB protoplasts with a silencing vector (GUN4_RNAi) and restoring its expression by transforming HYB protoplasts with a complementation vector (GUN4 ${ }^{\mathrm{CE}}$; Figure 7A).

The transcription of OsGUN4 and the 8 PRX genes was significantly affected by light intensity and OsGUN4 genotype (Supplementary Table 10). The native WT OsGUN4 promoter was used for the construct of both vectors, hence

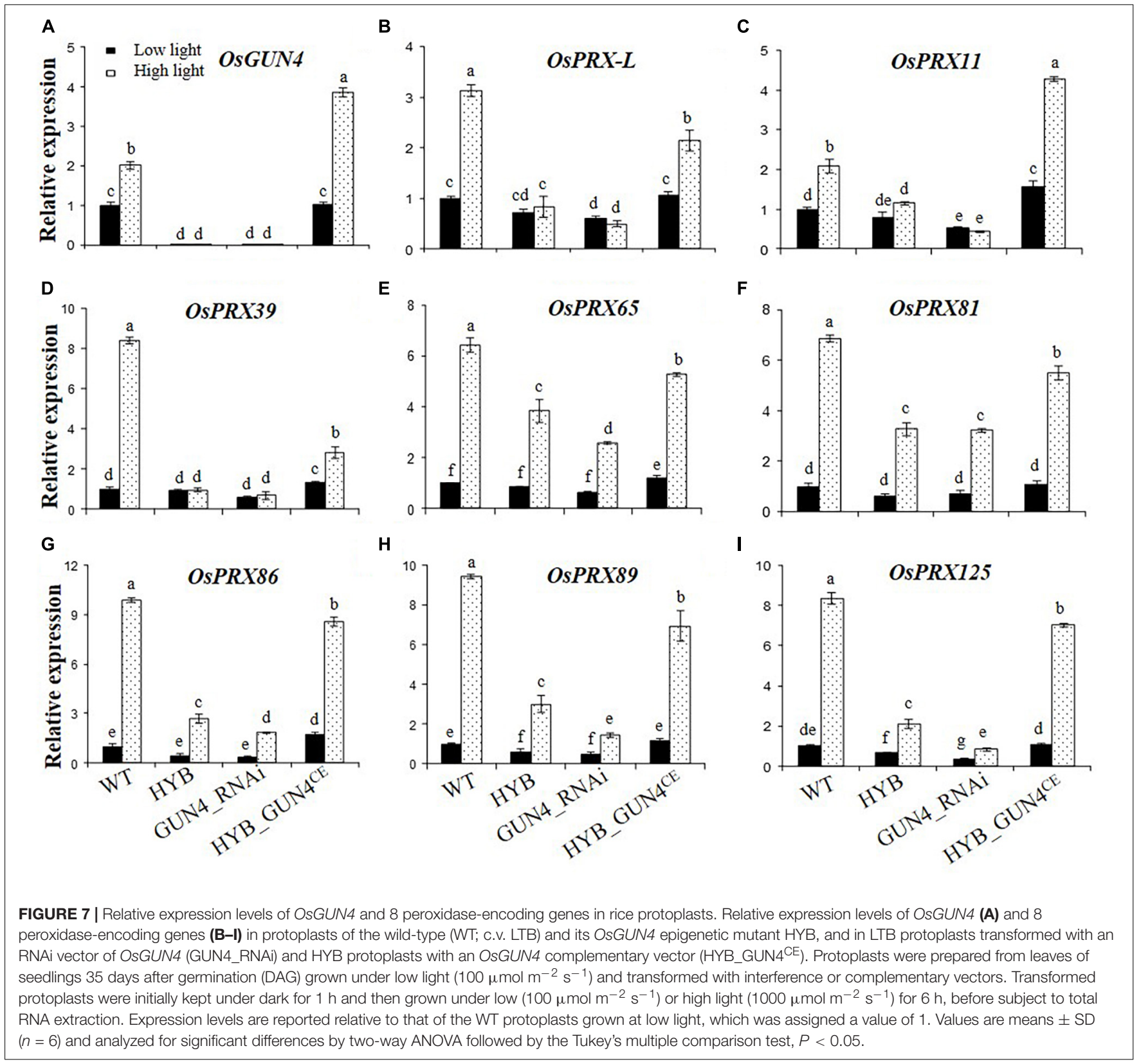


OsGUN4 expression in HYB protoplasts transformed with the complementation vector was also responsive to HL. In WT protoplasts transformed with OsGUN4-RNAi, transcription of OsGUN4 was downregulated to the level similar to that in HYB at both LL and HL, while HYB protoplasts transformed with $\mathrm{GUN} 4{ }^{\mathrm{CE}}$, the expression level of OsGUN4 was restored to nearly the same level of WT at LL, and to higher levels at HL (Figure 7A).

In both WT and $\mathrm{HYB} \_\mathrm{GUN} 4{ }^{\mathrm{CE}}$ protoplasts, transcription of all the 8 PRX genes was significantly increased after $\mathrm{HL}$ treatment. In HYB protoplasts transformed with $\mathrm{GUN} 4{ }^{\mathrm{CE}}$, the transcription level of $O s P R X$ genes was similar to or significantly higher than that of WT at LL, but did not restore to the same level as in the WT except OsPRX11 at HL (Figures 7B-I). Thus, the transcriptional response of PRX genes to light fluence shift was restored to different degrees in OsGUN4-complemented HYB protoplasts.

In both HYB and GUN4_RNAi protoplasts, either no such increase (OsPRX-L, OsPRX11 and OsPRX39) or limited increase of transcription was observed for the other genes, similar to the pattern observed in seedlings. In LTB protoplasts transformed with the interference vector, the expression level of OsPRXs was reduced to $10-44 \%$ that of untransformed protoplasts and was very similar to that observed in HYB, their response to HL was either abolished (OsPRX-L, OsPRX 11 and OsPRX39, Figures 7B-D) or significantly repressed (OsPRX65, OsPRX 81 and $O s P R X 86, O s P R X 89$ and $O s P R X 125$, Figures 7E-I).
These results demonstrate that the protoplast expression system worked well for interference and complementation of OsGUN4 mutation, and that there is a cause-and-effect relationship between OsGUN4 mutation and altered expression and response of the $8 P R X$ genes to a light fluence shift and $\mathrm{H}_{2} \mathrm{O}_{2}$ treatment.

\section{Effect of OsGUN4 and OsPRX39 Expression on ROS Accumulation in Protoplasts}

To examine whether OsGUN4 complementation and silencing affect ROS accumulation, the total ROS level in various types of protoplast was measured. Under LL conditions, no significant differences were observed among protoplasts with different OsGUN4 genotypes, however, significant differences were observed after transferred to HL conditions (Figure 8A and Supplementary Table 10). Consistent with the results in seedlings (Figure 4A), a significantly greater total ROS level was observed in HYB protoplasts than its WT parent (Figure 8A). Importantly, the total ROS level was increased in GUN4_RNAi LTB protoplasts (to a level even significantly higher than that of HYB), while the total ROS level was reduced to the level of the WT in GUN4 complemented HYB protoplasts $\left(\right.$ gun $4^{e p i}$ GUN4 $\left.{ }^{\mathrm{CE}}\right)$.

To further examine the effect of repression of PRX genes on ROS accumulation, we overexpressed OsPRX39 in HYB

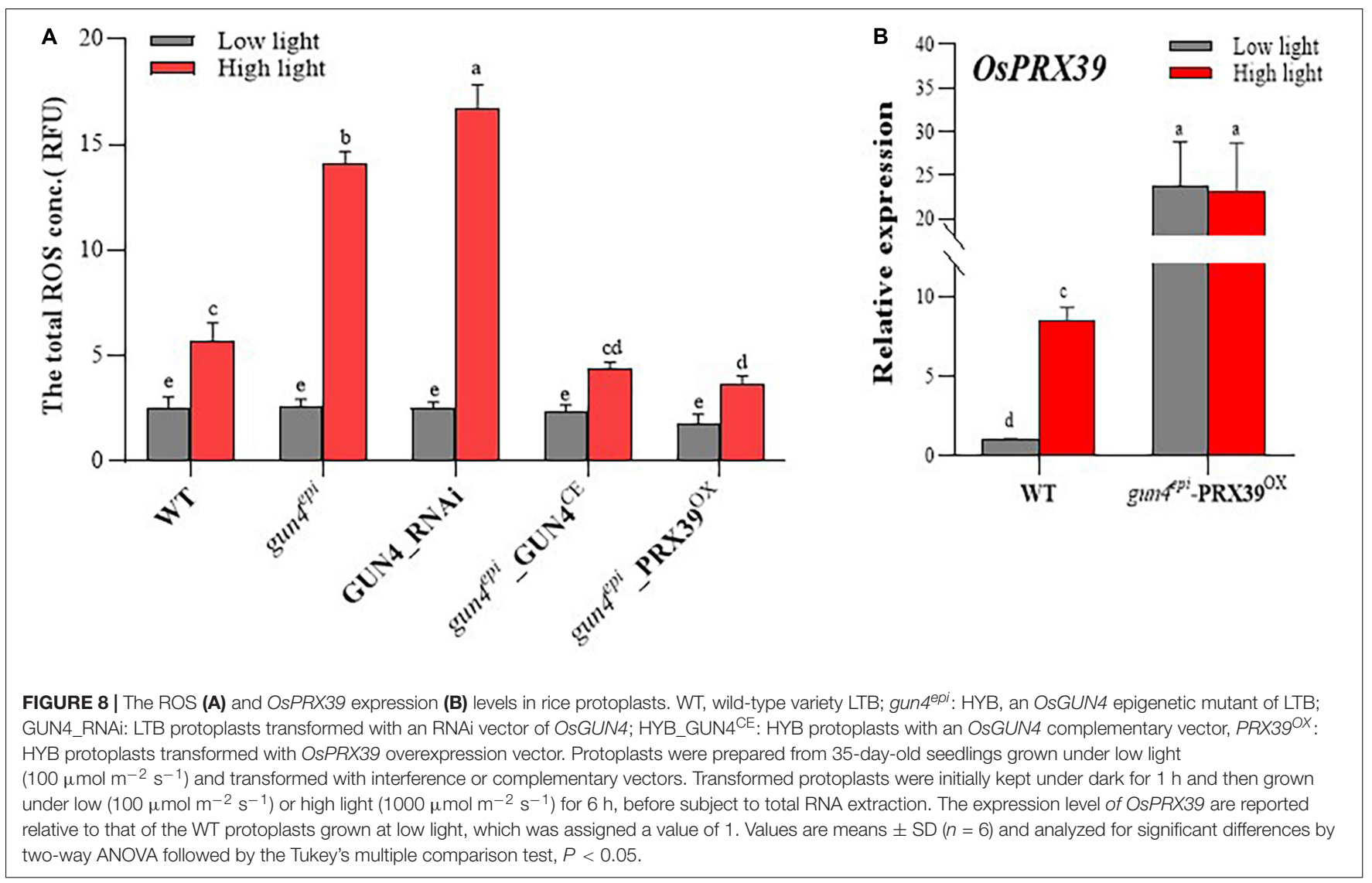


protoplasts $\left(g u n 4^{e p i}-P R X 39^{O X}\right)$. The OsPRX39 expression level in gun $4^{e p i}$ PRX39 $9^{O X}$ protoplasts was high under both LL and HL conditions because it was driven by the Ubi promoter, and it showed a 2.73-fold increase as compared to that of WT under HL (Figure 8B and Supplementary Table 10). The total ROS level in $g u n 4^{e p i}$ PRX39OX protoplasts was significantly reduced compared to the HYB protoplasts, and was even significantly lower that the WT LTB (Figure 8B).

\section{DISCUSSION}

The present study revealed that OsGUN4 expression is light- and oxidative-stress responsive (Figure 1) and its complete knockout conferred a lethal phenotype (Supplementary Figure 2), suggesting OsGUN4 is indispensable for seed germination and seedling growth in rice. Up to 10 times ROS that of WT was accumulated in completely knocked out mutants, which might explain its lethal effect. We identified 8 PRX genes that were significantly downregulated in the OsGUN4 mutant HYB and demonstrated that their expression was responsive to $\mathrm{HL}$ and $\mathrm{H}_{2} \mathrm{O}_{2}$ treatment in WT rice. However, the transcriptional response of the $8 \mathrm{PRX}$ genes to $\mathrm{HL}$ and $\mathrm{H}_{2} \mathrm{O}_{2}$ treatment were either abolished or severely impaired in the OsGUN4 mutants. Furthermore, we showed that there is a cause-and-effect relationship between OsGUN4 mutation and altered expression and response of the $8 P R X$ genes to a light fluence shift in a protoplast expression system. Put together, we demonstrated that transcription of OsGUN4 is regulated by oxidative stress and its response plays a critical role in ROS scavenging rather than ROS production, and we argue that the transcriptional regulation of the $8 P R X$ genes is facilitated by OsGUN4 via an as yet unknown signaling pathway in rice.

\section{OsGUN4 Transcription Is Responsive to Oxidative Stress}

We have made three significant observations:

(i) The expression of OsGUN4 is sensitive to light intensity and oxidative stress (Figures 1, 7A). In photosynthetic organisms, the major sources of ROS production are PSI and PSII reaction centers (Asada, 2006; Møller et al., 2007), which is consistent with our observation that significantly more ROS is accumulated in WT seedlings grown under $\mathrm{HL}$ than LL conditions (Figure 7A). As we previously reported, there is an anti-oxidative element (ARE) in the promoter of OsGUN4 (Li et al., 2014a), hence the observation that its transcription is responsive to oxidative stress was expected. Here, we further argue that the response of OsGUN4 transcription to light intensity and oxidative stress might be governed by the same mechanism or by overlapping mechanisms.

(ii) OsGUN4 transcription was low at night and increased rapidly at daybreak, and showed oscillation during the daytime (Figure 1A). The low expression at night is understandable because, at night, no photosynthesis takes place hence the ROS level also is low, while photosynthesis is active during the light period and the ROS level consequently increases. It is well-known that photosynthesis is reduced at midday in many crop plants including wheat (Sawada, 1978), rice (Ishihara and Saito, 1987), cotton (Pettigrew et al., 1990), maize (Tadashi and Theodore, 1999), and citrus (Hu et al., 2007). In a previous study, we observed the midday photoinhibition under natural field condition in the WT variety LTB (but not in HYB), with significantly reduced values of Fv/Fm and $\phi$ PSIIaround $1200 \mathrm{~h}$ (Wu et al., 2007). The underlying mechanism leading to midday depression of photosynthesis has been investigated from various aspects and at different levels, such as its relation to lightinduced chlorophyll fluorescence changes (Wang et al., 2021) and chloroplast positioning (Maai et al., 2020), however, it is not yet fully understood. We observed a decrease in OsGUN4 expression around midday in both WT varieties Nipponbare and LTB grown under HL conditions (Figure 1A), surprisingly similar to the reported midday depression of photosynthesis in LTB (Wu et al., 2007) and other varieties (Ishihara and Saito, 1987). It would be interesting to study whether depressed photosynthesis leads to a reduction in ROS content and subsequently to a downregulation of OsGUN4 transcription, or vice versa.

(iii) We observed a significant increase in total ROS content in OsGUN4 mutants. It is very interesting to note that while expression of OsGUN4 is responsive to ROS in WT LTB, its mutation caused elevated ROS accumulation in HYB. Elevated accumulation could result from either increased production or reduced scavenging of ROS. Because the overall photosynthetic activity in LTB is known to be higher than in HTB than in HYB, particularly before the midday depression of photosynthesis (Wu et al., 2007), and there was indeed a lower ${ }^{1} \mathrm{O}_{2}$ content, particularly under HL conditions (Figure 1C), therefore we argue that the it is very likely that the ROS scavenging capacity is impaired due to OsGUN4 mutations.

\section{ROS Accumulation in OsGUN4 Mutations May Result From Repression of PRX Genes}

Plant cells deploy a delicate system to prevent or limit ROS production (Holt et al., 2004) and exquisite antioxidant system to scavenge excessive ROS in chloroplast (Gupta et al., 2015) via non-enzymatic (Havaux et al., 2007) and enzymatic reactions (Dietz, 2016).

In the present study, we identified 8 peroxidase encoding genes that were downregulated in HYB as compared with LTB, and further demonstrated that their transcriptional response to oxidative stress was abolished or repressed in OsGUN4 mutant seedlings (Figure 6). Of particular importance is that the transcriptional repression of the 8 PRX genes was also observed in the mutant $\mathrm{F}_{2}$ seedlings, because it demonstrates that the differences between HYB and LTB is very likely due to the OsGUN4 mutation. We further established the cause-andeffect relationship between OsGUN4 mutation and its repression effect on transcription by silencing and complementing OsGUN4 in protoplasts and determination of expression of the 8 PRX genes (Figure 7).

Peroxidases are important ROS scavengers in plants. It is therefore predictable that OsGUN4 mutations such as downregulation (gun $4^{e p i}$ ) and knockout (gun4-1) would 
significantly impair the ROS scavenging system and result in ROS accumulation. Consistent with this prediction, the content of total ROS and $\mathrm{H}_{2} \mathrm{O}_{2}$ was significantly higher in the mutants than the WT, particularly under HL conditions (Figures 4A,B, 8).

The $g u n 4^{e p i}$ allele dramatically downregulates its transcription, but would still retain a very low level of OsGUN4 protein, while the gun4-1 allele is expected to completely abolish its translation, which means that it would produce no OsGUN4 protein. The expected different mutational effects on OsGUN4 expression was indeed further manifested at both the biochemical and phenotypical level. The gun4-1/gun4-1 seeds could not germinate normally and conferred early death (Supplementary Figure 2), which is very likely due to the very high ROS accumulation $\mathrm{t}$ (Figure 4A). Moreover, significantly

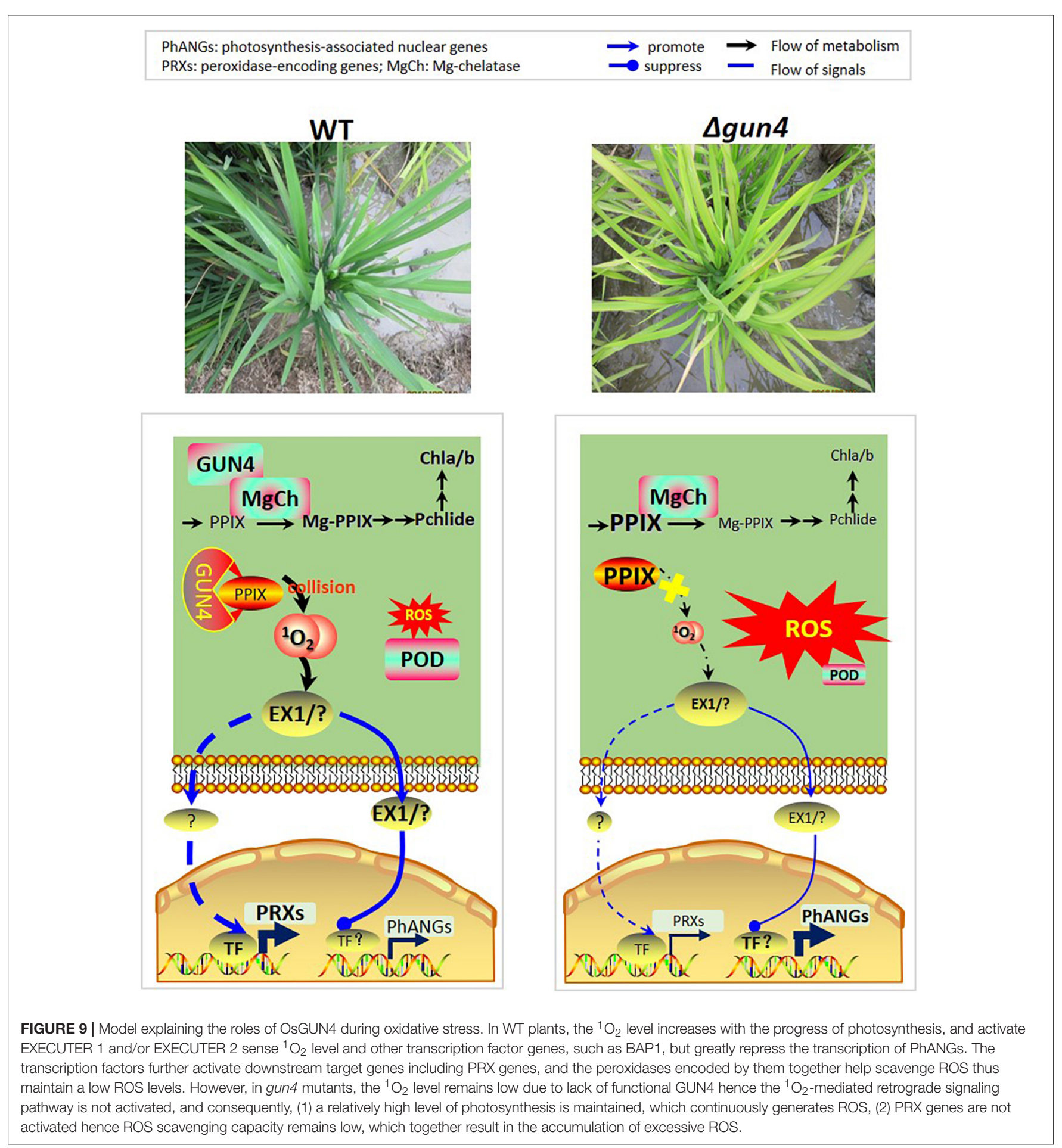


more ROS and $\mathrm{H}_{2} \mathrm{O}_{2}$ were accumulated in gun $4^{\text {epi }} /$ gun4-1 seedlings than in $g u n 4^{e p i} / g u n 4^{e p i}$ ones (Figures 4A,B), further supports the more severe effect of gun4-1. The different effects on ROS accumulation were well manifested on seedling growth: gun $4^{e p i} / g u n 4^{e p i}$ grew much better than gun $4^{e p i} / g u n 4-1$ seedlings (Figure 2).

\section{The Role of OsGUN4 in Retrograde Signaling and Regulation of PRX Genes, a Hypothesis}

Genomes Uncoupled 4 was identified as a regulator of chlorophyll synthesis and intracellular signaling in Arabidopsis (Larkin et al., 2003; Larkin, 2016). However, most of yet limited number of studies on GUN4 and its orthologs have been focusing on its regulatory function in tetrapyrrole biosynthesis, with only a few studies examined its role and mechanism in retrograde signaling.

In Chlamydomonas reinhardtii, Brzezowski et al. (2014) demonstrated that light-or dark-grown gun4 mutant accumulates high levels of PPIX, and fails to downregulate mRNA levels of the tetrapyrrole biosynthesis and the photosynthesis-associated nuclear genes (PhANGs). While they proposed that GUN4 functions in 'shielding' PPIX, and most likely Mg-PPIX, by reducing reactivity with $\mathrm{O}_{2}$, and further suggested that "GUN4 seems to be involved in sensing elevated levels of these photoreactive tetrapyrrole intermediates, and contributing to ${ }^{1} \mathrm{O}_{2}$-mediated retrograde signaling." However, Tabrizi et al. (2016) argued for the opposite mechanism in that GUN4-PPIX is a ${ }^{1} \mathrm{O}_{2}$ generator and proposed that the light-dependent ${ }^{1} \mathrm{O}_{2}$ generation from GUN4-PPIX is the first step in retrograde signaling from the chloroplast to the nucleus. While the two studies suggested quite different roles of GUN4 in ${ }^{1} \mathrm{O}_{2}$ generation, they suggested the same route of retrograde signaling, that is, EXECUTER 1 and/or EXECUTER 2 sense ${ }^{1} \mathrm{O}_{2}$ level and regulate transcription of PhANGs.

In rice, we previously observed that downregulation of OsGUN4 results in deregulated transcription of PhANGs and altered tetrapyrrole biosynthesis, as well as decreased ${ }^{1} \mathrm{O}_{2}$ production and down-regulated ${ }^{1} \mathrm{O}_{2}$-dependent retrograde signaling ( $\mathrm{Li}$ et al., 2017). In the present study, we observed the same concentration changes of PPIX, Mg-PPIX and pchlide (Figures 3A-C) and ${ }^{1} \mathrm{O}_{2}$ (Figure 4D) in both of $g u n 4^{e p i} / g u n 4^{e p i}$ and gun $4^{e p i} / g u n 4-1$ seedlings as in HYB. These results are consistent with the findings that OsGUN4 plays a role by promoting ${ }^{1} \mathrm{O}_{2}$ generation and its deficiency is expected to impair ${ }^{1} \mathrm{O}_{2}$-dependent retrograde signaling.

Regarding the role of GUN4 in retrograde signaling, it has so far limited to the ${ }^{1} \mathrm{O}_{2}$-dependent, EXECUTER 1 and/or EXECUTER 2 mediated transcription regulation of PhANGs. Our present study for the first time extended it to the regulation of PRX genes (Figure 9). While further studies are needed to establish the regulatory chain from GUN4 to PRX genes, we here propose that regulation of genes responsive for ROS scavenging, such as the PRX genes studied in the present study, is an essential function of the retrograde signaling pathway. We further envision the following working module: In WT plants, the ${ }^{1} \mathrm{O}_{2}$ level increases with the progress of photosynthesis, and activate EXECUTER 1 and/or EXECUTER 2 sense ${ }^{1} \mathrm{O}_{2}$ level and other transcription factor (TF) genes, such as BAP1. Some of the activated TFs repress the transcription of PhANGs after photosynthesis reaches to a high level, for example late morning, such that the photosynthesis is prohibited at midday. On the other side, some of the TFs activate genes including PRX genes, and the peroxidases encoded by them together help scavenge ROS thus maintain a low ROS level. However, in gun4 mutants, the ${ }^{1} \mathrm{O}_{2}$ level remains low due to lack of functional GUN4 hence the ${ }^{1} \mathrm{O}_{2}$-mediated retrograde signaling pathway is not activated, and consequently, (1) a relatively high level of photosynthesis is maintained, which continuously generates ROS, (2) PRX genes are not activated hence ROS scavenging capacity remains low, which together result in the accumulation of excessive ROS (Figure 9). More studies are needed to validate our hypothesis and reveal the players leading to the activation of PRX genes downstream the ${ }^{1} \mathrm{O}_{2}$-mediated signaling pathway.

\section{CONCLUSION}

We here demonstrate that OsGUN4 is indispensable for rice seed germination and seedling growth, its deficiency results in inactivation of the ${ }^{1} \mathrm{O}_{2}$ mediated signaling pathway. Consequently, the expression of PhANGs is deregulated and that of PRX genes is not activated, which together result in significant ROS accumulation in OsGUN4 mutants.

\section{DATA AVAILABILITY STATEMENT}

The original contributions presented in the study are publicly available. This data can be found here: NCBI repository, https: //www.ncbi.nlm.nih.gov/bioproject/PRJNA715818.

\section{ETHICS STATEMENT}

The authors declare that the experiments were performed in compliance with the current laws of China.

\section{AUTHOR CONTRIBUTIONS}

Q-YS conceived the study and finished the final version. $\mathrm{R}-\mathrm{QL}$ and $\mathrm{MJ}$ carried out the experimental analysis and data analysis. R-QL finished the first draft. IM and J-ZH revised the manuscript. All authors contributed to the article and approved the submitted version.

\section{FUNDING}

This work is funded by grants from the National Key Research and Development Program of China (2016YFD0102103), 
Natural Foundation of Anhui Province (1908085QC103), and by Funding of Introduction and Stability for Talents supported by Anhui Agricultural University (yj201839). IM was supported by a travel grant from the 111 Plan project.

\section{REFERENCES}

Adhikari, N. D., Froehlich, J. E., Strand, D. D., Buck, S. M., Kramer, D. M., and Larkin, R. M. (2011). GUN4-porphyrin complexes bind the ChlH/GUN5 subunit of mg-chelatase and promote chlorophyll biosynthesis in Arabidopsis. Plant Cell 23, 1449-1467. doi: 10.1105/tpc.110.082503

Asada, K. (1999). The water-water cycle in chloroplasts: Scavenging of active oxygens and dissipation of excess photons. Annu. Rev. Plant Biol. 50, 601-639.

Asada, K. (2006). Production and scavenging of reactive oxygen species in chloroplasts and their functions. Plant Physiol. 141, 391-396.

Bart, R., Chern, M., Park, C. J., Bartley, L., and Ronald, P. (2006). A novel system for gene silencing using siRNAs in rice leaf and stem-derived protoplasts. Plant Methods 2:13. doi: 10.1186/1746-4811-2-13

Brzezowski, P., Schlicke, H., Richter, A., Dent, R. M., Niyogi, K. K., and Grimm, B. (2014). The GUN4 protein plays a regulatory role in tetrapyrrole biosynthesis and chloroplast-to-nucleus signaling in Chlamydomonas reinhardtii. Plant J. 79, 285-298. doi: 10.1111/tpj.12560

Chen, X., Hua, P., Xiao, W., Long, W., Lin, R., and Lin, L. (2015). Crystal structures of gun4 in complex with porphyrins. Mol. Plant 8, 1125-1127. doi: 10.1016/j. molp.2015.04.013

Davison, P. A., Schubert, H. L., Reid, J. D., Iorg, C. D., Heroux, A., Hill, C. P., et al. (2005). Structural and biochemical characterization of Gun4 suggests a mechanism for its role in chlorophyll biosynthesis. Biochemistry 44, 7603-7612. doi: 10.1021/bi050240x

Dietz, K. J. (2016). Thiol-based peroxidases and ascorbate peroxidases: why plants rely on multiple peroxidase systems in the photosynthesizing chloroplast? Mol. Cells 39:20. doi: 10.14348/molcells.2016.2324

Dietz, K. J., and Hell, R. (2015). Thiol switches in redox regulation of chloroplasts: balancing redox state, metabolism and oxidative stress. Biol. Chem. 396, 483-494.

Engvall, E., and Perlmann, P. (1971). Enzyme-linked immunosorbent assay (elisa). quantitative assay of immunoglobulin g. Immunochemistry 8, 871-874.

Formighieri, C., Ceol, M., Bonente, G., Rochaix, J. D., and Bassi, R. (2012). Retrograde signaling and photoprotection in a gun 4 mutant of Chlamydomonas reinhardtii. Mol. Plant 5, 1242-1262. doi: 10.1093/mp/sss051

Gupta, D. K., Palma, J. M., and Corpas, F. J. (2015). "Production sites of reactive oxygen species (ROS) in organelles from plant cells," in Reactive Oxygen Species and Oxidative Damage in Plants Under Stress, eds D. K. Gupta, F. J. Corpas, and J. M. Palma (Salmon Tower Building, NY: Springer International Publishing), $1-22$.

Havaux, M., Dall'osto, L., and Bassi, R. (2007). Zeaxanthin has enhanced antioxidant capacity with respect to all other xanthophylls in Arabidopsis leaves and functions independent of binding to PSII antennae. Plant Physiol. 145, 1506-1520. doi: 10.1104/pp.107.108480

Hideg, É, Barta, C., Kálai, T., Vass, I., Hideg, K., and Asada, K. (2002). Detection of singlet oxygen and superoxide with fluorescent sensors in leaves under stress by photoinhibition or UV radiation. Plant Cell Physiol. 43, 1154-1164.

Holt, N. E., Fleming, G. R., and Niyogi, K. K. (2004). Toward an understanding of the mechanism of nonphotochemical quenching in green plants. Biochemistry 43, 8281-8289. doi: 10.1021/bi0494020

Hu, L. M., Xia, R. X., Xiao, Z. Y., Huang, R. H., Tan, M. L., Wang, M. Y., et al. (2007). Reduced leaf photosynthesis at midday in citrus leaves growing under field or screenhouse conditions. J. Hortic. Sci. Biotech. 82, 387-392. doi: 10. 1080/14620316.2007.11512248

Ishihara, K., and Saito, K. (1987). Diurnal course of photosynthesis, transpiration, and diffusive conductance in the single leaf of rice plants grown in the paddy field under submerged condition. Jpn. J Crop Sci. 56, 8-17. doi: 10.1626/jcs.56.8

Jiang, M., Liu, Y. H., Li, R. Q., Zheng, Y. C., Fu, H. W., Tan, Y. Y., et al. (2019). A suppressor mutation partially reverts the xantha trait via lowered methylation

\section{SUPPLEMENTARY MATERIAL}

The Supplementary Material for this article can be found online at: https://www.frontiersin.org/articles/10.3389/fpls.2021. 682453/full\#supplementary-material

in the promoter of genomes uncoupled 4 in rice. Front. Plant Sci. 10:1003. doi: 10.3389/fpls.2019.01003

Joo, J. H., Wang, S., Chen, J. G., Jones, A. M., and Fedoroff, N. V. (2005). Different signaling and cell death roles of heterotrimeric $G$ protein alpha and beta subunits in the Arabidopsis oxidative stress response to ozone. Plant Cell 17, 957-970. doi: 10.1105/tpc.104.029603

Koski, V. M., and Smith, J. H. (1948). The isolation and spectral absorption properties of protochlorophyll from etiolated barley seedlings. J. Am. Chem. Soc. 70, 3558-3562. doi: 10.1021/ja01191a006

Laloi, C., Apel, K., and Danon, A. (2004). Reactive oxygen signalling: the latest news. Curr. Opin. Plant Biol. 7, 323-328. doi: 10.1016/j.pbi.2004.03.005

Larkin, R. M. (2016). Tetrapyrrole signaling in plants. Front. Plant Sci. 7:1586. doi: $10.3389 /$ fpls.2016.01586

Larkin, R. M., Alonso, J. M., Ecker, J. R., and Chory, J. (2003). GUN4, a regulator of chlorophyll synthesis and intracellular signaling. Science 299, 902-906.

Li, R. Q., Huang, J. Z., Zhao, H. J., Fu, H. W., Li, Y. F., Liu, G. Z., et al. (2014a). A down-regulated epi-allele of the genomes uncoupled 4 gene generates a xantha marker trait in rice. Theor. Appl. Genet. 127, 491-501. doi: 10.1007/s00122014-2393-9

Li, R. Q., Jiang, M., Liu, Y. H., Zheng, Y. C., Huang, J. Z., Wu, J. M., et al. (2017). The xantha marker trait is associated with altered tetrapyrrole biosynthesis and deregulated transcription of PhANGs in rice. Front. Plant Sci. 8:901. doi: 10.3389/fpls.2017.00901

Li, W. X., Huang, J. Z., Zhao, H. J., Tan, Y. Y., Cui, H. R., Poirier, Y., et al. (2014b). Production of low phytic acid rice by hairpin RNA- and artificial microRNA-mediated silencing of OsMIK in seeds. Plant Cell Tiss. Organ Cult. $119,15-25$.

Livak, K. J., and Schmittgen, T. D. (2001). Analysis of relative gene expression data using real-time quantitative pcr and the 2(-delta delta $\mathrm{c}(\mathrm{t})$ ) method. Methods 25, 402-408. doi: 10.1006/meth.2001.1262

Maai, E., Nishimura, K., Takisawa, R., and Nakazaki, T. (2020). Diurnal changes in chloroplast positioning and photosynthetic traits of C-4 grass finger millet. Plant Pro. Sci. 23, 477-489. doi: 10.1080/1343943x.2020.1758171

Mochizuki, N., Tanaka, R., Tanaka, A., Masuda, T., and Nagatani, A. (2008). The steady-state level of Mg-Protoporphyrin IX is not a determinant of plastid-tonucleus signaling in Arabidopsis. Proc. Natl Acad. Sci. U.S.A. 105, 15184-15189. doi: 10.1073/pnas.0803245105

Møller, I. M., Jensen, P. E., and Hansson, A. (2007). Oxidative modifications to cellular components in plants. Annu. Rev. Plant Biol. 58, 459-481. doi: 10.1146/ annurev.arplant.58.032806.103946

Murashige, T., and Skoog, F. (1962). A revised medium for rapid growth and bioassays with tobacco tissue culture. Physiol. Plantarum 15, 473-496. doi: 10.1111/j.1399-3054.1962.tb08052.x

Papenbrock, J., and Grimm, B. (2001). Regulatory network of tetrapyrrole biosynthesis studies of intracellular signalling involved in metabolic and developmental control of plastids. Planta 213, 667-681.

Peter, E., and Grimm, B. (2009). GUN4 is required for posttranslational control of plant tetrapyrrole biosynthesis. Mol. Plant 2, 1198-1210. doi: 10.1093/mp/ ssp072

Pettigrew, W. T., Hesketh, J. D., Peters, D. B., and Woolley, J. T. (1990). A vapor pressure deficit on crop canopy photosynthesis. Photosynthesis Res. 24, 27-34. doi: $10.1007 /$ bf00032641

Richter, A. S., Hochheuser, C., Fufezan, C., Heinze, L., Kuhnert, F., and Grimm, B. (2016). Phosphorylation of genomes uncoupled 4 alters stimulation of $\mathrm{Mg}$ chelatase activity in angiosperms. Plant Physiol. 172, 1578-1595. doi: 10.1104/ pp.16.01036

Rokebul Anower, M., Peel, M. D., Mott, I. W., and Wu, Y. (2017). Physiological processes associated with salinity tolerance in an alfalfa half-sib family. J. Agr. Crop Sci. 203, 506-518. doi: 10.1111/jac.12221 
Sawada, S. I. (1978). Midday depression of photosynthesis in wheat seedlings. J. Crop Sci. 47, 18-24.

Sobotka, R., Dühring, U., Komenda, J., Peter, E., Gardian, Z., Tichy, M., et al. (2008). Importance of the cyanobacterial GUN4 protein for chlorophyll metabolism and assembly of photosynthetic complexes. J. Biol. Chem. 283, 25794-257802. doi: 10.1074/jbc.M803787200

Strand, Å, Asami, T., Alonso, J., Ecker, J. R., and Chory, J. (2003). Chloroplast to nucleus communication triggered by accumulation of $\mathrm{Mg}$-protoporphyrinix. Nature 421, 79-83. doi: 10.1038/nature01204

Susek, R. E., Ausubel, F. M., and Chory, J. (1993). Signal transduction mutants of Arabidopsis uncouple nuclear $\mathrm{Cab}$ and Rbcs gene expression from chloroplast development. Cell 74, 787-799. doi: 10.1016/0092-8674(93)90459-4

Tabrizi, S. T., Sawicki, A., Zhou, S., Luo, M., and Willows, R. D. (2016). GUN4protoporphyrin ix is a singlet oxygen generator with consequences for plastid retrograde signaling. J. Biol. Chem. 291, 8978-8984. doi: 10.1074/jbc.C116. 719989

Tadashi, H., and Theodore, C. H. (1999). Some characteristics of reduced leaf photosynthesis at midday in maize growing in the field. Field Crops Res. 62, 53-62. doi: 10.1016/s0378-4290(99)00005-2

Tan, Y. Y., Yu, X. M., Shu, Q. Y., Zhang, H. L., Wang, S. G., Yuan, F. J., et al. (2016). Development of an HRM-based, safe and high-throughput genotyping system for two low phytic acid mutations in soybean. Mol. Breeding 36, 101-109.

Trapnell, C., Roberts, A., Goff, L., Pertea, G., Kim, D., Kelley, D. R., et al. (2012). Differential gene and transcript expression analysis of RNA-seq experiments with TopHat and Cufflinks. Nat. Protoc. 7, 562-578. doi: 10.1038/nprot.2012.016

Verdecia, M. A., Larkin, R. M., Ferrer, J. L., Riek, R., Chory, J., and Noel, J. P. (2005). Structure of the Mg-chelatase cofactor GUN4 reveals a novel handshaped fold for porphyrin binding. Med. Sci. 21, 903-904. doi: 10.1051/medsci/ 20052111903
Wang, N., Suomalainen, J., Bartholomeus, H., Kooistra, L., Masiliunas, D., and Clevers, J. (2021). Diurnal variation of sun-induced chlorophyll fluorescence of agricultural crops observed from a point-based spectrometer on a UAV. Int. J. Appl. Earth Obs. 96, 102276. doi: 10.1016/j.jag.2020.10 2276

Wu, L., Shen, S., Wang, R., and Shu, Q. (2007). The diurnal variation of photosynthesis of a xantha mutant in rice. J. Nuclear Agric. Sci. 21, 425-429.

Zhang, Y., Su, J., Duan, S., Ao, Y., Dai, J., Liu, J., et al. (2011). A highly efficient rice green tissue protoplast system for transient gene expression and studying light/chloroplast-related processes. Plant Methods 7:30. doi: 10.1186/17464811-7-30

Zhou, S., Sawicki, A., Willows, R. D., and Luo, M. (2012). C-terminal residues of Oryza sativa GUN4 are required for the activation of the ChlH subunit of magnesium chelatase in chlorophyll synthesis. FEBS Lett. 586, 205-210. doi: 10.1016/j.febslet.2011.12.026

Zhou, X. S., Shen, S. Q., Wu, D. X., Sun, J. W., and Shu, Q. Y. (2006). Introduction of a xantha mutation for testing and increasing varietal purity in hybrid rice. Field Crops Res. 96, 71-79.

Conflict of Interest: The authors declare that the research was conducted in the absence of any commercial or financial relationships that could be construed as a potential conflict of interest.

Copyright (C) 2021 Li, Jiang, Huang, Møller and Shu. This is an open-access article distributed under the terms of the Creative Commons Attribution License (CC BY). The use, distribution or reproduction in other forums is permitted, provided the original author(s) and the copyright owner(s) are credited and that the original publication in this journal is cited, in accordance with accepted academic practice. No use, distribution or reproduction is permitted which does not comply with these terms. 